\title{
TENSOR PRODUCTS OF KIRILLOV-RESHETIKHIN MODULES AND FUSION PRODUCTS
}

\author{
KATSUYUKI NAOI
}

\begin{abstract}
We study the classical limit of a tensor product of Kirillov-Reshetikhin modules over a quantum loop algebra, and show that it is realized from the classical limits of the tensor factors using the notion of fusion products. In the process of the proof, we also give defining relations of the fusion product of the (graded) classical limits of Kirillov-Reshetikhin modules.
\end{abstract}

\section{INTRODUCTION}

Let $\mathfrak{g}$ be a complex simple Lie algebra, and $\mathbf{L} \mathfrak{g}=\mathfrak{g} \otimes \mathbb{C}\left[t, t^{-1}\right]$ the associated loop algebra. The theory of finite-dimensional representations over the quantum loop algebra $U_{q}(\mathbf{L} \mathfrak{g})$ has been intensively studied from various viewpoints over the past two decades. Kirillov-Reshetikhin modules (KR modules, for short) are a subclass of finitedimensional simple $U_{q}(\mathbf{L g})$-modules, and have attracted a particular interest because of their rich combinatorial structures and several applications to mathematical physics (see [KR87, HKO ${ }^{+99}$, Nak03, Her06, OS08, CH10, Ked11, Nao13a] and references therein).

One approach which has been used to explore the structure of a finite-dimensional $U_{q}(\mathbf{L} \mathfrak{g})$-module is to study its classical limit, or graded limit. A classical limit is an $\mathbf{L} \mathfrak{g}$-module which is obtained from a $U_{q}(\mathbf{L} \mathfrak{g})$-module by specializing the quantum parameter $q$ to 1 . In addition, in many interesting cases, by restricting a classical limit to the current algebra $\mathfrak{g}[t]=\mathfrak{g} \otimes \mathbb{C}[t] \subseteq \mathbf{L} \mathfrak{g}$ and taking a pull-back with respect to an automorphism, we obtain a graded $\mathfrak{g}[t]$-module, which is called the graded limit. After the pioneering work of Chari and Pressley CP01, several formulas of characters and multiplicities are obtained for KR modules Cha01, CM06] and their generalizations called minimal affinizations [Mou10, Nao13b, Nao14, LN16], by analyzing their classical or graded limits.

Moreover, graded limits are important as well in view of the theory of finitedimensional graded $\mathfrak{g}[t]$-modules, since they provide nontrivial and probably interesting such modules. (Though the original motivation to study finite-dimensional graded $\mathfrak{g}[t]$ modules was mainly an application to the theory of $U_{q}(\mathbf{L} \mathfrak{g})$-modules, they are now also of independent interest, since they have connections with problems arising in mathematical physics such as the $X=M$ conjecture [AK07, DFK08, Nao12a], and theory of symmetric functions such as Macdonald polynomials [CI15].) In fact, in [CV15] the authors have constructed a short exact sequence of $\mathfrak{g}[t]$-modules as a graded limit ana$\log$ of the $T$-system, which is a distinguished exact sequence of $U_{q}(\mathbf{L} \mathfrak{g})$-modules (see [Her06]). This is an interesting example of the study of graded $\mathfrak{g}[t]$-modules, motivated by the notion of graded limits. Since the process of obtaining a graded limit from a classical limit is elementary, we will mainly focus on classical limits in this paper. 
One difficulty in studying classical limits is the noncommutativity between the operations of tensor product and taking the limit. Namely, the limit of a tensor product of $U_{q}(\mathbf{L} \mathfrak{g})$-modules is not necessarily isomorphic to the tensor product of their limits. There are several examples (which will be listed below) which suggest that, to obtain the limit, we have to replace (some of) tensor products with fusion products. Here fusion products are graded analogs of tensor products of graded $\mathfrak{g}[t]$-modules introduced by Feigin and Loktev in FL99]. The purpose of this paper is to show the statement for an arbitrary tensor product of KR modules.

Let $I$ be the index set of simple roots of $\mathfrak{g}$. We denote a KR module by $W_{q}^{i, \ell}(a)$, which is parametrized by an index $i \in I$, a positive integer $\ell$ and a rational function $a \in \mathbb{C}(q)$. The graded limit of $W_{q}^{i, \ell}(a)$ is denoted by $W^{i, \ell}$ (which does not depend on $a)$. We now state the main theorem of this paper (Theorem 3.1).

Theorem A. Assume that a given tensor product $W_{q}^{i_{1}, \ell_{1}}\left(a_{1}\right) \otimes \cdots \otimes W_{q}^{i_{p}, \ell_{p}}\left(a_{p}\right)$ of $K R$ modules has a classical limit (for the precise conditions, see Theorem 3.1).

(i) If $a_{1}(1)=\cdots=a_{p}(1)=c \in \mathbb{C}^{\times}$, then the following isomorphism of $\mathfrak{g}[t]$-modules holds:

$$
\overline{W_{q}^{i_{1}, \ell_{1}}\left(a_{1}\right) \otimes \cdots \otimes W_{q}^{i_{p}, \ell_{p}}\left(a_{p}\right)} \cong \varphi_{c}^{*}\left(W^{i_{1}, \ell_{1}} * \cdots * W^{i_{p}, \ell_{p}}\right) .
$$

Here the left-hand side is the classical limit, $*$ denotes the fusion product, and $\varphi_{c}^{*}$ the pull-back with respect to the automorphism $\varphi_{c}$ of $\mathfrak{g}[t]$ defined by $\varphi_{c}(x \otimes f(t))=x \otimes f(t+c)$.

(ii) In the general case, the following isomorphism of $\mathfrak{g}[t]$-modules holds:

$$
\overline{W_{q}^{i_{1}, \ell_{1}}\left(a_{1}\right) \otimes \cdots \otimes W_{q}^{i_{p}, \ell_{p}}\left(a_{p}\right)} \cong \bigotimes_{c \in \mathbb{C}^{\times}} \varphi_{c}^{*}\left(\underset{k ; a_{k}(1)=c}{*} W^{i_{k}, \ell_{k}}\right),
$$

where $* W^{i_{k}, \ell_{k}}$ denotes the fusion product of $W^{i_{k}, \ell_{k}}$ 's.

We remark that the assertion (i) implies that the graded limit of the tensor product is isomorphic to the fusion product of their graded limits. There are several special cases where the theorem has already been proved, which we list here.

- In the case where $\ell_{1}=\cdots=\ell_{p}=1$, the result follows from CL06, FL07, Nao12b.

- In the case of type $A$ with $i_{1}=\cdots=i_{p}$ and $a_{1}=\cdots=a_{p}$, the result is proved in BP15].

- For a special class of tensor products appearing in the $T$-system, the result follows from [CV15].

Our proof is valid for arbitrary $\mathfrak{g}$ and an arbitrary tensor product of KR modules, even if it is reducible (as long as the classical limit exists). It should be mentioned that a special class of tensor products in type $A$, whose factors are not necessarily isomorphic to KR modules, is treated in BCM15.

The proof of Theorem A is carried out in two steps. In the first step, we give defining relations of the fusion product of the graded limits of KR modules. Let us mention the precise statement. Let $\varpi_{i}(i \in I)$ be the fundamental weights. Let $\mathfrak{g}=\mathfrak{n}_{+} \oplus \mathfrak{h} \oplus \mathfrak{n}_{-}$ be a triangular decomposition, and denote the Chevalley generators by $e_{i}, h_{i}, f_{i}(i \in I)$. We show the following theorem (Theorem 3.3), which is a generalization of the result for $\mathfrak{g}=\mathfrak{s l}_{2}$ in FF02, CV15. 
Theorem B. A fusion product $W^{i_{1}, \ell_{1}} * \cdots * W^{i_{p}, \ell_{p}}$ is isomorphic to the $\mathfrak{g}[t]$-module generated by a single vector $v$ with relations

$$
\begin{gathered}
\mathfrak{n}_{+}[t] v=0, \quad(h \otimes f(t)) v=f(0)\langle h, \lambda\rangle v \quad \text { for } h \in \mathfrak{h}, f(t) \in \mathbb{C}[t], \\
\left(F_{i}(z)^{r}\right)_{s} v=0 \text { for } i \in I, r>0, s<-\sum_{k ; i_{k}=i} \min \left\{r, \ell_{k}\right\},
\end{gathered}
$$

where we set $\lambda=\sum_{k=1}^{p} \ell_{k} \varpi_{i_{k}}$, and $\left(F_{i}(z)^{r}\right)_{s} \in U(\mathfrak{g}[t])$ denotes the coefficient of $z^{s}$ in the $r$-th power of $F_{i}(z)=\sum_{k=0}^{\infty}\left(f_{i} \otimes t^{k}\right) z^{-k-1} \in U(\mathfrak{g}[t])\left[\left[z^{-1}\right]\right]$.

This theorem answers affirmatively the question raised in [FH14, Introduction] (see Remark 3.4 (a) of the present paper). Note that the presentation is more refined than that given in Nao15 in type $A$. The main tool we use for the proof is the functional realization of the dual of $U\left(\mathbf{L n}_{-}\right)$, which has been introduced in FS94 and further developed in $\mathrm{FKL}^{+} 02$, AKS06, AK07]. In particular, in AKS06, AK07 the authors also study fusion products of $W^{i, \ell}$ s using the functional realization (though with a different motivation), which inspired our proof.

Using Theorem B, we can reduce the proof of Theorem A to the case $\mathfrak{g}=\mathfrak{s l}_{2}$ (the key to this is the fact that the relations in Theorem B essentially contain only root vectors corresponding to simple roots). Then in the second step, we show the case of $\mathfrak{s l}_{2}$ independently. This is an outline of the proof of Theorem A.

The plan of this paper is as follows. In Section 2, we give preliminary definitions and basic results. In Section 3, we reduce the proof of Theorem A to the case $\mathfrak{g}=\mathfrak{s l}_{2}$ and Theorem B. In Section 1 we prove Theorem B, but we postpone some proofs of assertions concerning with the functional realization to Appendix A. In Section 5, we show Theorem A for $\mathfrak{g}=\mathfrak{s l}_{2}$. Finally in Appendix A, we give proofs postponed in Section 1 .

\section{Preliminaries}

2.1. Lie algebras. Let $\widehat{C}=\left(c_{i j}\right)_{0 \leq i, j \leq n}$ be a Cartan matrix of nontwisted affine type, and assume that the indices are ordered as Kac90, Section 4.8]. Let $C=\left(c_{i j}\right)_{1 \leq i, j \leq n}$, which is a Cartan matrix of finite type. Set $I=\{1, \ldots, n\}$ and $\widehat{I}=\{0\} \cup I$. Fix a diagonal matrix $\widehat{D}=\operatorname{diag}\left(d_{0}, \ldots, d_{n}\right)$ such that $d_{i} \in \mathbb{Z}_{>0}$ and $\widehat{D} \widehat{C}$ is symmetric.

Let $\mathfrak{g}$ be the complex simple Lie algebra associated with $C$, and fix a triangular decomposition $\mathfrak{g}=\mathfrak{n}_{+} \oplus \mathfrak{h} \oplus \mathfrak{n}_{-}$. Let $\alpha_{i} \in \mathfrak{h}^{*}$ be the simple roots, and $\varpi_{i} \in \mathfrak{h}^{*}$ the fundamental weights $(i \in I)$. Let $P$ and $Q$ be the weight and root lattices respectively, and set

$$
P^{+}=\sum_{i \in I} \mathbb{Z}_{\geq 0} \varpi_{i}, \quad Q^{+}=\sum_{i \in I} \mathbb{Z}_{\geq 0} \alpha_{i} .
$$

For $\gamma=\sum_{i} m_{i} \alpha_{i} \in Q^{+}$, let ht $(\gamma)=\sum_{i} m_{i}$ denote the height of $\gamma$. Denote by $R$ the root system, and by $R^{+}$the set of positive roots. For each root $\alpha \in R$ denote by $h_{\alpha} \in \mathfrak{h}$ its coroot. Let $W$ be the Weyl group with simple reflections $\left\{s_{i} \mid i \in I\right\}$. Let $\theta \in R^{+}$ be the highest root.

Denote by $\mathfrak{g}_{\alpha}(\alpha \in R)$ the root spaces, and for each $\alpha \in R^{+}$fix vectors $e_{\alpha} \in \mathfrak{g}_{\alpha}$ and $f_{\alpha} \in \mathfrak{g}_{-\alpha}$ satisfying $\left[e_{\alpha}, f_{\alpha}\right]=h_{\alpha}$. We also use the notations $e_{i}=e_{\alpha_{i}}, f_{i}=f_{\alpha_{i}}, h_{i}=h_{\alpha_{i}}$. 
For $i \in I$, denote by $\mathfrak{s l}_{2, i}$ the Lie subalgebra of $\mathfrak{g}$ spanned by $e_{i}, f_{i}, h_{i}$. For $\lambda \in P^{+}$, denote by $V(\lambda)$ the finite-dimensional simple $\mathfrak{g}$-module with highest weight $\lambda$.

Let $\varpi_{i}^{\vee} \in \mathfrak{h}(i \in I)$ be the fundamental coweights, and $P^{\vee}=\bigoplus_{i \in I} \mathbb{Z}_{i}^{\vee} \subseteq \mathfrak{h}$ the coweight lattice. The group

$$
\widetilde{W}=W \ltimes P^{\vee}
$$

is called the extended affine Weyl group. Write $w$ for $(w, 0) \in \widetilde{W}$, and $t_{x}$ for (id, $\left.x\right) \in \widetilde{W}$. Let $\widehat{Q}=Q \oplus \mathbb{Z} \delta$ be the affine root lattice, where $\delta$ is the null root. $\widetilde{W}$ acts on $\widehat{Q}$ by

$$
w(\lambda+a \delta)=w(\lambda)+a \delta \text { and } t_{x}(\lambda+a \delta)=\lambda+(a-\langle x, \lambda\rangle) \delta,
$$

where $w \in W, x \in P^{\vee}, \lambda \in Q, a \in \mathbb{Z}$. Set $\alpha_{0}=\delta-\theta \in \widehat{Q}$ and $s_{0}=s_{\theta} t_{-h_{\theta}} \in \widetilde{W}$, where $s_{\theta}$ is the reflection with respect to $\theta$. Let

$$
\widehat{W}=\left\langle s_{i} \mid i \in \widehat{I}\right\rangle \subseteq \widetilde{W}
$$

be the affine Weyl group. Denote by $\Gamma$ the subgroup of $\widetilde{W}$ consisting of the elements preserving the set $\left\{\alpha_{i} \mid i \in \widehat{I}\right\}$. Then $\Gamma$ also acts on the set $\widehat{I}$ as permutations, and is identified with a subgroup of the Dynkin diagram automorphisms of $\widehat{C}$. It follows that $\widetilde{W}=\Gamma \ltimes \widehat{W}$, where $\tau \in \Gamma$ acts on $\widehat{W}$ so that

$$
\tau s_{i} \tau^{-1}=s_{\tau(i)} \text { for } i \in \widehat{I} .
$$

Given a complex Lie algebra $\mathfrak{a}$, its loop algebra $\mathbf{L} \mathfrak{a}$ is defined by the tensor product $\mathfrak{a} \otimes \mathbb{C}\left[t, t^{-1}\right]$, whose Lie algebra structure is given by

$$
[x \otimes f(t), y \otimes g(t)]=[x, y] \otimes f(t) g(t) .
$$

Denote by $\mathfrak{a}[t]$ and $t^{k} \mathfrak{a}[t]$ for $k \in \mathbb{Z}_{>0}$ the Lie subalgebras $\mathfrak{a} \otimes \mathbb{C}[t]$ and $\mathfrak{a} \otimes t^{k} \mathbb{C}[t]$, respectively. The Lie algebra $\mathfrak{a}[t]$ is called the current algebra associated with $\mathfrak{a}$. For $c \in \mathbb{C}$, define a Lie algebra automorphism $\varphi_{c}=\varphi_{c}^{\mathfrak{a}}$ on $\mathbf{L a}$ by

$$
\varphi_{c}(x \otimes f(t))=x \otimes f(t+c) \text { for } x \in \mathfrak{a}, f(t) \in \mathbb{C}[t] .
$$

2.2. Fusion product. Here we recall the notion of fusion products introduced in [FL99]. Note that the degree grading on $\mathbb{C}[t]$ induces $\mathbb{Z}$-gradings on $\mathfrak{g}[t]$ and $U(\mathfrak{g}[t])$. Denote by $\mathfrak{g}[t]^{k}$ and $U(\mathfrak{g}[t])^{k}$ the subspaces with degree $k$. We say a $\mathfrak{g}[t]$-module $M$ is graded if a $\mathbb{Z}$-grading $M=\bigoplus_{k \in \mathbb{Z}} M^{k}$ is given and $\mathfrak{g}[t]^{k} M^{\ell} \subseteq M^{\ell+k}$ holds for all $k, \ell \in \mathbb{Z}$.

Let $M_{1}, \ldots, M_{p}$ be cyclic finite-dimensional graded $\mathfrak{g}[t]$-modules with respective generators $v_{1}, \ldots, v_{p}$, and $c_{1}, \ldots, c_{p}$ pairwise distinct complex numbers. For each $1 \leq j \leq p$, define a $\mathfrak{g}[t]$-module $\left(M_{j}\right)_{c_{j}}$ by the pull-back $\varphi_{c_{j}}^{*} M_{j}$, and set $M=\left(M_{1}\right)_{c_{1}} \otimes \cdots \otimes\left(M_{p}\right)_{c_{p}}$. It follows from [FL99 that $M$ is generated by the single vector $v_{1} \otimes \cdots \otimes v_{p}$ as a $\mathfrak{g}[t]$-module. The module $M$ is not graded, but a filtration is defined on $M$ by

$$
F^{\leq k}(M)=\sum_{\ell \leq k} U(\mathfrak{g}[t])^{\ell}\left(v_{1} \otimes \cdots \otimes v_{p}\right) .
$$

The associated graded space $\bigoplus_{k} F^{\leq k}(M) / F^{\leq k-1}(M)$ has a natural graded $\mathfrak{g}[t]$-module structure, which is called the fusion product of $M_{1}, \ldots, M_{p}$ and denoted by

$$
M_{1} * M_{2} * \cdots * M_{p}
$$

Although the definition depends on the parameters $c_{i}$ and the generators $v_{i}$, we omit them for ease of notation. All fusion products appearing below are known not to depend 
on the parameters up to isomorphism, and the choices of the generators will be clear from the context. Note that the fusion product does not depend on the order of the factors up to isomorphism.

2.3. Quantum loop algebras. Let $q$ be an indeterminate. For $\ell \in \mathbb{Z}, s, s^{\prime} \in \mathbb{Z}_{\geq 0}$ with $s \geq s^{\prime}$, set

$$
[\ell]_{q}=\frac{q^{\ell}-q^{-\ell}}{q-q^{-1}}, \quad[s]_{q} !=[s]_{q}[s-1]_{q} \cdots[1]_{q}, \quad\left[\begin{array}{c}
s \\
s^{\prime}
\end{array}\right]_{q}=\frac{[s]_{q} !}{\left[s^{\prime}\right]_{q} !\left[s-s^{\prime}\right]_{q} !} .
$$

Write $q_{i}=q^{d_{i}}$ for $i \in \widehat{I}$. The quantum loop algebra $U_{q}(\mathbf{L g})$ is a $\mathbb{C}(q)$-algebra generated by $k_{i}^{ \pm 1}, X_{i}^{ \pm}(i \in \widehat{I})$ with relations

$$
\begin{array}{r}
k_{i} k_{i}^{-1}=k_{i}^{-1} k_{i}=1, \quad k_{i} k_{j}=k_{j} k_{i}, \quad k_{i} X_{j}^{ \pm} k_{i}^{-1}=q_{i}^{ \pm c_{i j}} X_{j}^{ \pm}, \quad k_{\delta}=1, \\
{\left[X_{i}^{+}, X_{j}^{-}\right]=\delta_{i j} \frac{k_{i}-k_{i}^{-1}}{q_{i}-q_{i}^{-1}}, \quad \sum_{k=0}^{s}(-1)^{k}\left[\begin{array}{c}
s \\
k
\end{array}\right]_{q_{i}}\left(X_{i}^{ \pm}\right)^{k} X_{j}^{ \pm}\left(X_{i}^{ \pm}\right)^{s-k}=0(i \neq j),}
\end{array}
$$

where $k_{\delta}=\prod_{i \in \widehat{I}} k_{i}^{a_{i}}$ with $\delta=\sum a_{i} \alpha_{i}$, and $s=1-c_{i j}$. Let $U_{q}(\mathfrak{g}) \subseteq U_{q}(\mathbf{L g})$ be the $\mathbb{C}(q)$ subalgebra generated by $k_{i}^{ \pm 1}, X_{i}^{ \pm}(i \in I)$, which is the quantized enveloping algebra associated with $\mathfrak{g}$. The algebra $U_{q}(\mathbf{L} \mathfrak{g})$ has a Hopf algebra structure [Lus93, CP94, and the comultiplication is given by

$$
\Delta\left(X_{i}^{+}\right)=X_{i}^{+} \otimes 1+k_{i} \otimes X_{i}^{+}, \quad \Delta\left(X_{i}^{-}\right)=X_{i}^{-} \otimes k_{i}^{-1}+1 \otimes X_{i}^{-}, \quad \Delta\left(k_{i}\right)=k_{i} \otimes k_{i} .
$$

For $i \in \widehat{I}$, let $T_{i}=T_{i, 1}^{\prime \prime}$ be the algebra automorphism of $U_{q}(\mathbf{L g})$ in [Lus93, Chapter 37]. Given $w=\tau w^{\prime} \in \widetilde{W}$ with $\tau \in \Gamma$ and $w^{\prime} \in \widehat{W}$, choose a reduced expression $w^{\prime}=s_{i_{1}} \cdots s_{i_{k}}$ and set $T_{w}=T_{\tau} T_{i_{1}} \cdots T_{i_{k}}$, where $T_{\tau}$ is the algebra automorphism on $U_{q}(\mathbf{L} \mathfrak{g})$ naturally induced from the diagram automorphism $\tau$. The automorphism $T_{w}$ does not depend on the choice of the expression. For $x \in P^{\vee}$, write $T_{x}=T_{t_{x}}$ for ease of notation.

There is another presentation of $U_{q}(\mathbf{L} \mathfrak{g})$ Dri87, Bec94]. In this presentation, $U_{q}(\mathbf{L} \mathfrak{g})$ is a $\mathbb{C}(q)$-algebra with generators

$$
x_{i, r}^{ \pm}(i \in I, r \in \mathbb{Z}), \quad k_{i}^{ \pm 1}(i \in I), \quad h_{i, m}(i \in I, m \in \mathbb{Z} \backslash\{0\})
$$

and the following defining relations $\left(i, j \in I, r, r^{\prime} \in \mathbb{Z}, m, m^{\prime} \in \mathbb{Z} \backslash\{0\}\right)$ :

$$
\begin{gathered}
k_{i} k_{i}^{-1}=k_{i}^{-1} k_{i}=1, \quad\left[k_{i}, k_{j}\right]=\left[k_{i}, h_{j, m}\right]=\left[h_{i, m}, h_{j, m^{\prime}}\right]=0, \\
k_{i} x_{j, r}^{ \pm} k_{i}^{-1}=q_{i}^{ \pm c_{i j}} x_{j, r}^{ \pm}, \quad\left[h_{i, m}, x_{j, r}^{ \pm}\right]= \pm \frac{1}{m}\left[m c_{i j}\right]_{q_{i}} x_{j, r+m}^{ \pm}, \\
{\left[x_{i, r}^{+}, x_{j, r^{\prime}}^{-}\right]=\delta_{i j} \frac{\phi_{i, r+r^{\prime}}^{+}-\phi_{i, r+r^{\prime}}^{-}}{q_{i}-q_{i}^{-1}},} \\
x_{i, r+1}^{ \pm} x_{j, r^{\prime}}^{ \pm}-q_{i}^{ \pm c_{i j}} x_{j, r^{\prime}}^{ \pm} x_{i, r+1}^{ \pm}=q_{i}^{ \pm c_{i j}} x_{i, r}^{ \pm} x_{j, r^{\prime}+1}^{ \pm}-x_{j, r^{\prime}+1}^{ \pm} x_{i, r}^{ \pm}, \\
\sum_{\sigma \in \mathfrak{S}_{s}} \sum_{k=0}^{s}(-1)^{k}\left[\begin{array}{c}
s \\
k
\end{array}\right]_{q_{i}} x_{i, r_{\sigma(1)}}^{ \pm} \cdots x_{i, r_{\sigma(k)}}^{ \pm} x_{j, r^{\prime}}^{ \pm} x_{i, r_{\sigma(k+1)}}^{ \pm} \cdots x_{i, r_{\sigma(s)}}^{ \pm}=0 \quad(i \neq j)
\end{gathered}
$$


for all sequences of integers $r_{1}, \ldots, r_{s}$, where $s=1-c_{i j}, \mathfrak{S}_{s}$ is the symmetric group on $s$ letters, and $\phi_{i, r}^{ \pm}$'s are determined by equating coefficients of powers of $u$ in the formula

$$
\sum_{r=0}^{\infty} \phi_{i, \pm r}^{ \pm} u^{ \pm r}=k_{i}^{ \pm 1} \exp \left( \pm\left(q_{i}-q_{i}^{-1}\right) \sum_{m=1}^{\infty} h_{i, \pm m} u^{ \pm m}\right),
$$

and $\phi_{i, \mp r}^{ \pm}=0$ for $r>0$. Let $o: I \rightarrow\{ \pm 1\}$ be a map satisfying $o(i)=-o(j)$ whenever $c_{i j}<0$. Then we have

$$
x_{i, m}^{ \pm}=o(i)^{m} T_{\varpi_{i}^{\vee}}^{\mp m}\left(X_{i}^{ \pm}\right) .
$$

Let $U_{q}\left(\mathbf{L n}_{ \pm}\right)$be the subalgebras of $U_{q}(\mathbf{L} \mathfrak{g})$ generated by $\left\{x_{i, r}^{ \pm} \mid i \in I, r \in \mathbb{Z}\right\}$, and $U_{q}(\mathbf{L h})$ the subalgebra generated by $\left\{k_{i}^{ \pm 1}, h_{i, m} \mid i \in I, m \in \mathbb{Z} \backslash\{0\}\right\}$. It is easily proved from the defining relations that

$$
U_{q}(\mathbf{L} \mathfrak{g})=U_{q}\left(\mathbf{L n}_{-}\right) U_{q}(\mathbf{L} \mathfrak{h}) U_{q}\left(\mathbf{L n}_{+}\right)
$$

A $U_{q}(\mathfrak{g})$-module $M$ is said to be of type 1 if

$$
M=\bigoplus_{\lambda \in P} M_{\lambda}, \quad M_{\lambda}=\left\{v \in M \mid k_{i}^{ \pm 1} v=q_{i}^{ \pm\left\langle h_{i}, \lambda\right\rangle} v\right\} .
$$

In this paper, we will only consider $U_{q}(\mathfrak{g})$-modules (and $U_{q}(\mathbf{L} \mathfrak{g})$-modules) of type 1 . For $\lambda \in P^{+}$, denote by $V_{q}(\lambda)$ the simple $U_{q}(\mathfrak{g})$-module (of type 1) with highest weight $\lambda$.

Let $P_{q}^{+}$denote the monoid (under coordinate-wise multiplication) of $I$-tuples of polynomials $\boldsymbol{\pi}=\left(\boldsymbol{\pi}_{1}(u), \ldots, \boldsymbol{\pi}_{n}(u)\right)$ such that each $\boldsymbol{\pi}_{i}(u)$ is expressed as

$$
\boldsymbol{\pi}_{i}(u)=\left(1-a_{1} u\right)\left(1-a_{2} u\right) \cdots\left(1-a_{k} u\right)
$$

for some $k \geq 0$ and $a_{j} \in \mathbb{C}(q)^{\times}$. Define a map wt: $P_{q}^{+} \rightarrow P^{+}$by

$$
\operatorname{wt}(\boldsymbol{\pi})=\sum_{i \in I}\left(\operatorname{deg} \boldsymbol{\pi}_{i}\right) \varpi_{i} .
$$

We say a $U_{q}(\mathbf{L g})$-module $V$ is $\ell$-highest weight with $\ell$-highest weight vector $v$ if it holds that

$$
x_{i, r}^{+} v=0 \text { for } i \in I, r \in \mathbb{Z}, \quad U_{q}(\mathbf{L} \mathfrak{h}) v=\mathbb{C}(q) v, \quad V=U_{q}(\mathbf{L g}) v .
$$

It follows from CP95 that for every $\pi \in P_{q}^{+}$, there exists a unique (up to isomorphism) simple finite-dimensional $\ell$-highest weight $U_{q}(\mathbf{L} \mathfrak{g})$-module, which we denote by $L_{q}(\boldsymbol{\pi})$, such that its $\ell$-highest weight vector $v_{\boldsymbol{\pi}}$ (which is unique up to a scalar multiplication) satisfies

$$
k_{i}^{ \pm 1} v_{\boldsymbol{\pi}}=q_{i}^{ \pm\left\langle h_{i}, \operatorname{wt}(\boldsymbol{\pi})\right\rangle} v_{\boldsymbol{\pi}}, \quad h_{i, m} v_{\boldsymbol{\pi}}=[m]_{q_{i}} d_{i, m} v_{\boldsymbol{\pi}} \text { for } i \in I, m \in \mathbb{Z} \backslash\{0\},
$$

where $d_{i, m} \in \mathbb{C}(q)$ are determined by the formula

$$
\exp \left(-\sum_{m=1}^{\infty} d_{i, \pm m} u^{m}\right)=\boldsymbol{\pi}_{i}^{ \pm}(u)
$$

Here $\boldsymbol{\pi}_{i}^{+}(u)=\boldsymbol{\pi}_{i}(u)$, and $\boldsymbol{\pi}_{i}^{-}(u)=\left(1-a_{1}^{-1} u\right) \cdots\left(1-a_{k}^{-1} u\right)$ if (2.3) holds. If $V$ is an $\ell$-highest weight module and its $\ell$-highest weight vector $v_{\boldsymbol{\pi}}$ satisfies (2.4), we say that the $\ell$-highest weight of $V$ is $\boldsymbol{\pi}$. The following lemma is a consequence of [FM01, Corollary 6.9]. 
Lemma 2.1. There exists a bijection $I \ni i \mapsto \bar{i} \in I$ and an integer $K$ such that, if we set $\boldsymbol{\pi}^{*}=\left(\boldsymbol{\pi}_{\bar{i}}\left(q^{K} u\right)\right)_{i \in I}$ for $\boldsymbol{\pi} \in P_{q}^{+}$, then the dual module $L_{q}(\boldsymbol{\pi})^{*}$ is isomorphic to $L_{q}\left(\boldsymbol{\pi}^{*}\right)$.

In this paper, we are mainly interested in the following special simple modules.

Definition 2.2. Given $i \in I, \ell \in \mathbb{Z}_{>0}$ and $a \in \mathbb{C}(q)$, define $\boldsymbol{\pi}_{i, \ell, a} \in P_{q}^{+}$by

$$
\left(\boldsymbol{\pi}_{i, \ell, a}\right)_{j}(u)= \begin{cases}\prod_{k=0}^{\ell-1}\left(1-a q_{i}^{2 k} u\right) & \text { if } j=i, \\ 1 & \text { otherwise. }\end{cases}
$$

The simple $U_{q}(\mathbf{L} \mathfrak{g})$-module $L_{q}\left(\boldsymbol{\pi}_{i, \ell, a}\right)$ is called the Kirillov-Reshetikhin module (KR module for short) associated with $i, \ell, a$, and denoted by $W_{q}^{i, \ell}(a)$.

We end this subsection by recalling the following lemma.

Lemma 2.3. (i) For $\boldsymbol{\pi}, \boldsymbol{\pi}^{\prime} \in P_{q}^{+}$, the simple $U_{q}(\mathbf{L g})$-module $L_{q}\left(\boldsymbol{\pi} \boldsymbol{\pi}^{\prime}\right)$ is isomorphic to a quotient of the $U_{q}(\mathbf{L} \mathfrak{g})$-submodule of $L_{q}(\boldsymbol{\pi}) \otimes L_{q}\left(\boldsymbol{\pi}^{\prime}\right)$ generated by the tensor product of $\ell$-highest weight vectors. In particular if $L_{q}(\boldsymbol{\pi}) \otimes L_{q}\left(\boldsymbol{\pi}^{\prime}\right)$ is simple, then we have

$$
L_{q}(\boldsymbol{\pi}) \otimes L_{q}\left(\boldsymbol{\pi}^{\prime}\right) \cong L_{q}\left(\boldsymbol{\pi}^{\prime}\right) \otimes L_{q}(\boldsymbol{\pi})
$$

as $U_{q}(\mathbf{L g})$-modules.

(ii) Let $W_{q}^{i_{1}, \ell_{1}}\left(a_{1}\right)$ and $W_{q}^{i_{2}, \ell_{2}}\left(a_{2}\right)$ be two KR modules, and assume that $a_{1} \notin q^{\mathbb{Z}} a_{2}$. Then we have

$$
W_{q}^{i_{1}, \ell_{1}}\left(a_{1}\right) \otimes W_{q}^{i_{2}, \ell_{2}}\left(a_{2}\right) \cong W_{q}^{i_{2}, \ell_{2}}\left(a_{2}\right) \otimes W_{q}^{i_{1}, \ell_{1}}\left(a_{1}\right) .
$$

Proof. The assertion (i) is proved in CP94, CP95]. It follows from Cha02, Theorem 6.1] and Lemma 2.1 that the module $W_{q}^{i_{1}, \ell_{1}}\left(a_{1}\right) \otimes W_{q}^{i_{2}, \ell_{2}}\left(a_{2}\right)$ in (ii) and its dual are both $\ell$-highest weight, and hence simple. Now the assertion (ii) follows from (i), and the proof is complete.

2.4. Subalgebras $\boldsymbol{U}_{\boldsymbol{q}, \boldsymbol{i}}$. For $i \in I$, let $U_{q, i}$ be the $\mathbb{C}(q)$-subalgebra of $U_{q}(\mathbf{L} \mathfrak{g})$ generated by $x_{i, r}^{ \pm}(r \in \mathbb{Z})$ and $k_{i}^{ \pm 1}$. Denote by $U_{q}\left(\mathbf{L s l}_{2}\right)_{d_{i}}$ the quantum loop algebra associated with $\widehat{C}=\left(\begin{array}{cc}2 & -2 \\ -2 & 2\end{array}\right)$ and $\widehat{D}=\left(\begin{array}{cc}d_{i} & 0 \\ 0 & d_{i}\end{array}\right)$. By Bec94, Proposition 3.8], there is a $\mathbb{C}(q)$-algebra isomorphism $\Psi_{i}: U_{q}\left(\mathbf{L s l}_{2}\right)_{d_{i}} \stackrel{\sim}{\rightarrow} U_{q, i}$ such that

$$
\begin{aligned}
& \Psi_{i}\left(k_{1}^{ \pm 1}\right)=k_{i}^{ \pm 1}, \quad \Psi_{i}\left(k_{0}^{ \pm 1}\right)=k_{i}^{\mp 1}, \quad \Psi_{i}\left(X_{1}^{ \pm}\right)=x_{i, 0}^{ \pm}, \\
& \Psi_{i}\left(X_{0}^{+}\right)=-o(i) k_{i}^{-1} x_{i, 1}^{-}, \quad \Psi_{i}\left(X_{0}^{-}\right)=-o(i) x_{i,-1}^{+} k_{i} .
\end{aligned}
$$

It should be remarked that $U_{q, i}$ is not a sub-coalgebra, and hence $\Psi_{i}$ is not a coalgebra isomorphism. The following lemma is needed later.

Lemma 2.4. Let $\boldsymbol{\pi}^{1}, \ldots, \boldsymbol{\pi}^{p}$ be a sequence of elements of $P_{q}^{+}$, and assume that $L_{q}\left(\boldsymbol{\pi}^{1}\right) \otimes$ $\cdots \otimes L_{q}\left(\boldsymbol{\pi}^{p}\right)$ is $\ell$-highest weight. Then the $U_{q, i}$-submodule

$$
U_{q, i}\left(v_{\boldsymbol{\pi}^{1}} \otimes \cdots \otimes v_{\boldsymbol{\pi}^{p}}\right) \subseteq L_{q}\left(\boldsymbol{\pi}^{1}\right) \otimes \cdots \otimes L_{q}\left(\boldsymbol{\pi}^{p}\right)
$$

generated by the tensor product of $\ell$-highest weight vectors is isomorphic, as a $U_{q}\left(\mathbf{L s l}_{2}\right)_{d_{i}}{ }^{-}$ module, to

$$
L_{q}\left(\boldsymbol{\pi}_{i}^{1}(u)\right) \otimes \cdots \otimes L_{q}\left(\boldsymbol{\pi}_{i}^{p}(u)\right)
$$


Proof. Let $\lambda=\sum_{k=1}^{p} \operatorname{wt}\left(\boldsymbol{\pi}^{k}\right) \in P^{+}$. Since $L_{q}\left(\boldsymbol{\pi}^{1}\right) \otimes \cdots \otimes L_{q}\left(\boldsymbol{\pi}^{p}\right)$ is $\ell$-highest weight, we easily see from (2.2) and the weight consideration that

$$
\begin{aligned}
U_{q, i}\left(v_{\boldsymbol{\pi}^{1}} \otimes \cdots \otimes v_{\boldsymbol{\pi}^{p}}\right) & =\bigoplus_{k \in \mathbb{Z}_{\geq 0}}\left(L_{q}\left(\boldsymbol{\pi}^{1}\right) \otimes \cdots \otimes L_{q}\left(\boldsymbol{\pi}^{p}\right)\right)_{\lambda-k \alpha_{i}} \\
& =\left(U_{q, i} v_{\boldsymbol{\pi}^{1}}\right) \otimes \cdots \otimes\left(U_{q, i} v_{\boldsymbol{\pi}^{p}}\right) .
\end{aligned}
$$

By CP96, Lemma 2.3], each $U_{q, i} v_{\boldsymbol{\pi}^{k}}$ is isomorphic to $L_{q}\left(\boldsymbol{\pi}_{i}^{k}(u)\right)$ as a $U_{q}\left(\mathbf{L s l}_{2}\right)_{d_{i}}$-module. Note that, the module (2.6) is defined through the coproduct of $U_{q}\left(\mathbf{L s l}_{2}\right)_{d_{i}}$, and hence it is not obvious that this is isomorphic to the module in (2.7), which is defined through the coproduct of $U_{q}(\mathbf{L} \mathfrak{g})$. However this is proved in [loc. cit., Lemma 2.2], and hence the lemma follows.

2.5. Classical limits. Let $\mathcal{A}$ be the local subring of $\mathbb{C}(q)$ defined by

$$
\mathcal{A}=\{f / g \mid f, g \in \mathbb{C}[q], g(1) \neq 0\} .
$$

An $\mathcal{A}$-lattice $L$ of a $\mathbb{C}(q)$-vector space $V$ is a free $\mathcal{A}$-submodule such that $V \cong \mathbb{C}(q) \otimes_{\mathcal{A}} L$. Let $U_{\mathcal{A}}(\mathbf{L g}) \subseteq U_{q}(\mathbf{L} \mathfrak{g})$ be the $\mathcal{A}$-subalgebra generated by $k_{i}^{ \pm 1}, x_{i, r}^{ \pm}(i \in I, r \in \mathbb{Z})$, and define $U_{\mathcal{A}}\left(\mathbf{L n}_{ \pm}\right) \subseteq U_{q}\left(\mathbf{L n}_{ \pm}\right), U_{\mathcal{A}}(\mathbf{L} \mathfrak{h}) \subseteq U_{q}(\mathbf{L} \mathfrak{h})$ and $U_{\mathcal{A}, i} \subseteq U_{q, i}(i \in I)$ by the $\mathcal{A}$ subalgebras generated by the given generators of the respective $\mathbb{C}(q)$-algebras. It is easily seen from the defining relations that $h_{i, m} \in U_{\mathcal{A}}(\mathbf{L g})$, and then it is proved that

$$
U_{\mathcal{A}}(\mathbf{L} \mathfrak{g})=U_{\mathcal{A}}\left(\mathbf{L} \mathfrak{n}_{-}\right) U_{\mathcal{A}}(\mathbf{L h}) U_{\mathcal{A}}\left(\mathbf{L n}_{+}\right)
$$

Lemma 2.5. The $\mathcal{A}$-subalgebra $U_{\mathcal{A}}(\mathbf{L} \mathfrak{g})$ coincides with the $\mathcal{A}$-subalgebra generated by $k_{i}^{ \pm 1}, X_{i}^{ \pm}(i \in \widehat{I})$. In particular, $U_{\mathcal{A}}(\mathbf{L} \mathfrak{g})$ is an $\mathcal{A}$-lattice [Lus90 and a sub-coalgebra.

Proof. Let $\widetilde{U}_{\mathcal{A}}(\mathbf{L g})$ denote the $\mathcal{A}$-subalgebra generated by $k_{i}^{ \pm 1}, X_{i}^{ \pm}(i \in \widehat{I})$. Since $T_{w}^{ \pm 1}$ $(w \in \widetilde{W})$ preserve $\widetilde{U}_{\mathcal{A}}(\mathbf{L g})$, the containment $U_{\mathcal{A}}(\mathbf{L} \mathfrak{g}) \subseteq \widetilde{U}_{\mathcal{A}}(\mathbf{L} \mathfrak{g})$ follows from (2.1). To show the opposite containment, we shall prove first that $T_{w}^{ \pm 1}(w \in \widetilde{W})$ also preserve $U_{\mathcal{A}}(\mathbf{L} \mathfrak{g})$, which is equivalent to that $T_{\varpi_{i}^{\vee}}^{ \pm 1}$ and $T_{i}^{ \pm 1}(i \in I)$ preserve $U_{\mathcal{A}}(\mathbf{L} \mathfrak{g})$ by Lus89, Lemma 2.8]. The former is easily proved from the fact $T_{\varpi_{i}^{\vee}}\left(x_{j, r}^{ \pm}\right)=x_{j, r}^{ \pm}(i \neq j)$ [Bec94, Corollary 3.2]. To show the latter, we need to prove that $T_{i}^{ \pm 1}\left(x_{j, r}^{+}\right) \in U_{\mathcal{A}}(\mathbf{L} \mathfrak{g})$ and $T_{i}^{ \pm 1}\left(x_{j, r}^{-}\right) \in U_{\mathcal{A}}(\mathbf{L} \mathfrak{g})$ for all $i, j \in I$ and $r \in \mathbb{Z}$. We prove the first assertion (the other is similarly proved). If $i \neq j$, then

$$
T_{i}^{ \pm 1}\left(x_{j, r}^{+}\right)=o(j)^{r} T_{i}^{ \pm 1} T_{\varpi_{j}^{\vee}}^{-r}\left(X_{j}^{+}\right)=o(j)^{r} T_{\varpi_{j}^{\vee}}^{-r} T_{i}^{ \pm 1}\left(X_{j}^{+}\right)
$$

(see [Lus89, Lemma 2.2]), which belongs to $U_{\mathcal{A}}(\mathbf{L g})$ since $T_{i}^{ \pm 1}\left(X_{j}^{+}\right)$is contained in the $\mathcal{A}$-subalgebra generated by $k_{a}^{ \pm 1}$ and $X_{a}^{ \pm}(a \in I)$. Assume that $i=j$, and consider the isomorphism $\Psi_{i}: U_{q}\left(\mathbf{L s l}_{2}\right)_{d_{i}} \rightarrow U_{q, i}$. By Bec94, Corollary 3.8], we have $\Psi_{i} \circ T_{1}=T_{i} \circ \Psi_{i}$ and $\Psi_{i} \circ T_{\varpi_{1}^{\vee}}=T_{\varpi_{i}^{\vee}} \circ \Psi_{i}$. Hence it follows that

$$
T_{i}^{ \pm 1}\left(x_{i, r}^{+}\right)=o(i)^{r} \Psi_{i}\left(T_{1}^{ \pm 1} T_{\varpi_{1}^{\vee}}^{-r}\left(X_{1}^{+}\right)\right) \in \Psi_{i}\left(\widetilde{U}_{\mathcal{A}}\left(\mathbf{L s l}_{2}\right)_{d_{i}}\right) .
$$

It is easily seen from (2.5) that $\Psi_{i}$ maps $\widetilde{U}_{\mathcal{A}}\left(\mathbf{L s l}_{2}\right)_{d_{i}}$ into $U_{\mathcal{A}}(\mathbf{L} \mathfrak{g})$, and hence we have $T_{i}^{ \pm 1}\left(x_{i, r}^{+}\right) \in U_{\mathcal{A}}(\mathbf{L} \mathfrak{g})$, as required. 
Let $w \in \widetilde{W}$ and $i \in I$ be such that $w\left(\alpha_{i}\right)=\alpha_{0}$. By Lus93, we have $T_{w}\left(X_{i}^{ \pm}\right)=$ $X_{0}^{ \pm}$, and hence $X_{0}^{ \pm} \in U_{\mathcal{A}}(\mathbf{L} \mathfrak{g})$ follows from the assertion proved above. This implies $U_{\mathcal{A}}(\mathbf{L} \mathfrak{g}) \supseteq \widetilde{U}_{\mathcal{A}}(\mathbf{L} \mathfrak{g})$, and the proof is complete.

Let $P_{\mathcal{A}}^{+}$be the submonoid of $P_{q}^{+}$consisting of $\boldsymbol{\pi}=\left(\boldsymbol{\pi}_{1}(u), \ldots, \boldsymbol{\pi}_{n}(u)\right)$ such that $\boldsymbol{\pi}_{i}^{ \pm}(u) \in \mathcal{A}[u]$ for all $i \in I$. The following lemma in simply-laced or classical types follows from CP01, Cha01, and the proof can be extended to general types. For completeness we give a more elementary proof here (in the papers cited above the assertion is proved over $\mathbb{C}\left[q, q^{-1}\right]$, and therefore the proof is more involved).

Lemma 2.6. Assume that $V$ is a finite-dimensional $\ell$-highest weight $U_{q}(\mathbf{L} \mathfrak{g})$-module with $\ell$-highest weight $\boldsymbol{\pi} \in P_{\mathcal{A}}^{+}$, and let $v_{\boldsymbol{\pi}}$ be an $\ell$-highest weight vector. Then the $U_{\mathcal{A}}(\mathbf{L} \mathfrak{g})$-submodule $L=U_{\mathcal{A}}(\mathbf{L} \mathfrak{g}) v_{\boldsymbol{\pi}} \subseteq V$ is an $\mathcal{A}$-lattice of $V$.

Proof. By (2.4) and (2.8), we have $L=U_{\mathcal{A}}\left(\mathbf{L n}_{-}\right) v_{\boldsymbol{\pi}}$. Let $N=\max \left\{\operatorname{deg} \boldsymbol{\pi}_{i}(u) \mid i \in I\right\}$. We first show the claim that any vector of the form

$$
x_{i_{1}, k_{1}}^{-} x_{i_{2}, k_{2}}^{-} \cdots x_{i_{p}, k_{p}}^{-} v_{\boldsymbol{\pi}}
$$

can be written as an $\mathcal{A}$-linear combination of vectors

$$
x_{i_{1}^{\prime}, k_{1}^{\prime}}^{-} x_{i_{2}^{\prime}, k_{2}^{\prime}}^{-} \cdots x_{i_{p}^{\prime}, k_{p}^{\prime}}^{-} v_{\boldsymbol{\pi}}, \quad\left|k_{j}^{\prime}\right|<N+p .
$$

We proceed by the induction on $p$. The case $p=1$ follows from CP01, Proposition 4.3]. Let $p>1$, and assume that $k_{1} \geq 0$ (the case $k_{1}<0$ is similarly proved). By the induction hypothesis we may assume that $\left|k_{j}\right|<N+p-1$ for $2 \leq j \leq p$. Then using the relation

$$
x_{i_{1}, k_{1}}^{-} x_{i_{2}, k_{2}}^{-}=q_{i_{1}}^{-c_{i_{1} i_{2}}} x_{i_{2}, k_{2}}^{-} x_{i_{1}, k_{1}}^{-}+q_{i_{1}}^{-c_{i_{1} i_{2}}} x_{i_{1}, k_{1}-1}^{-} x_{i_{2}, k_{2}+1}^{-}-x_{i_{2}, k_{2}+1}^{-} x_{i_{1}, k_{1}-1}^{-},
$$

and applying the induction hypothesis on $p$ again, we easily see that the claim is proved by the induction on $k_{1}$.

Since $V$ is finite-dimensional, vectors of the form (2.9) are 0 when $p$ is sufficiently large. Hence it follows from the claim that $L$ is finitely generated as an $\mathcal{A}$-module. Since $L$ is obviously torsion-free, the lemma follows.

Denote by ${ }^{-}: \mathcal{A} \rightarrow \mathbb{C}$ the $\mathbb{C}$-algebra homomorphism defined by $\bar{q}=1$. Given an $\mathcal{A}$-module $M$, denote by $M_{\mathbb{C}}$ the $\mathbb{C}$-vector space $\mathbb{C} \otimes_{\mathcal{A}} M$ where $\mathcal{A}$ acts on $\mathbb{C}$ via ${ }^{-}$. For an element $v \in M$, write $\bar{v}=1 \otimes v \in M_{\mathbb{C}} . U_{\mathcal{A}}(\mathbf{L g})_{\mathbb{C}}$ has a natural $\mathbb{C}$-algebra structure, and there exists a surjective $\mathbb{C}$-algebra homomorphism (see [CP94, Proposition 9.2.3])

$$
U_{\mathcal{A}}(\mathbf{L} \mathfrak{g})_{\mathbb{C}} \rightarrow U(\mathbf{L g}): \overline{x_{i, r}^{+}} \mapsto e_{i} \otimes t^{r}, \overline{x_{i, r}^{-}} \mapsto f_{i} \otimes t^{r}, \overline{k_{i}^{ \pm 1}} \mapsto 1, \overline{h_{i, m}} \mapsto h_{i} \otimes t^{m},
$$

whose kernel is the ideal of $U_{\mathcal{A}}(\mathbf{L g})_{\mathbb{C}}$ generated by $\overline{k_{i}}-1(i \in I)$. Assume that $V$ is a finite-dimensional $\ell$-highest weight $U_{q}(\mathbf{L} \mathfrak{g})$-module with $\ell$-highest weight $\boldsymbol{\pi} \in P_{\mathcal{A}}^{+}$and $\ell$-highest weight vector $v_{\boldsymbol{\pi}}$, and set $L=U_{\mathcal{A}}(\mathbf{L} \mathfrak{g}) v_{\boldsymbol{\pi}} \subseteq V$. Then through the algebra homomorphism $L_{\mathbb{C}}$ becomes an $\mathbf{L g}$-module, which is called the classical limit of $V$ and denoted by $\bar{V}$. By Lemma 2.6 we have $\operatorname{dim}_{\mathbb{C}(q)} V=\operatorname{dim}_{\mathbb{C}} \bar{V}$, and it is also easy to see that

$$
\left[V: V_{q}(\lambda)\right]_{U_{q}(\mathfrak{g})}=[\bar{V}: V(\lambda)]_{\mathfrak{g}}
$$


holds for all $\lambda \in P^{+}$(see Nao13b, Subsection 3.4], for example), where the left- and right-hand sides denote the multiplicities as $U_{q}(\mathfrak{g})$ - and $\mathfrak{g}$-modules, respectively.

The following $\mathfrak{g}[t]$-modules are introduced in [Cha01, CM06].

Definition 2.7. For $i \in I, \ell \in \mathbb{Z}_{>0}$ and $c \in \mathbb{C}$, let $W^{i, \ell}(c)$ be a $\mathfrak{g}[t]$-module generated by a vector $v=v_{i, \ell, c}$ with relations

$$
\begin{gathered}
\mathfrak{n}_{+}[t] v=0, \quad(h \otimes f(t)) v=\ell f(c)\left\langle h, \varpi_{i}\right\rangle v \text { for } h \in \mathfrak{h}, f(t) \in \mathbb{C}[t], \\
f_{i}^{\ell+1} v=\left(f_{i} \otimes(t-c)\right) v=0, \quad f_{j} v=0 \text { for } j \in I \backslash\{i\} .
\end{gathered}
$$

When $c=0$, we simply write $W^{i, \ell}=W^{i, \ell}(c)$.

Note that $\varphi_{c}^{*}\left(W^{i, \ell}\left(c^{\prime}\right)\right)=W^{i, \ell}\left(c+c^{\prime}\right)$. It is easily seen that, for sufficiently large $N$, $\mathfrak{g} \otimes(t-c)^{N} \mathbb{C}[t]$ acts trivially on $W^{i, \ell}(c)$. Hence when $c \neq 0$, by considering the Lie algebra homomorphism

$$
\mathbf{L} \mathfrak{g} \rightarrow \mathfrak{g} \otimes\left(\mathbb{C}[[t-c]] /(t-c)^{N} \mathbb{C}[[t-c]]\right) \cong \mathfrak{g} \otimes\left(\mathbb{C}[t] /(t-c)^{N} \mathbb{C}[t]\right)
$$

induced by the Taylor expansion, $W^{i, \ell}(c)$ is uniquely lifted to an $\mathbf{L g}$-module.

Proposition 2.8. Let $i \in I, \ell \in \mathbb{Z}_{>0}$ and $a \in \mathcal{A}^{\times}$. The classical limit $\overline{W_{q}^{i, \ell}(a)}$ of the KR module $W_{q}^{i, \ell}(a)$ is isomorphic to $W^{i, \ell}(\bar{a})$ as an $\mathbf{L g}$-module.

Proof. It is easy to check that the relations (2.10) with $\mathbb{C}[t]$ replaced by $\mathbb{C}\left[t, t^{-1}\right]$ are the defining relations of $W^{i, \ell}(c)(c \neq 0)$ as an $\mathbf{L} \mathfrak{g}$-module. Then the existence of a surjection $W^{i, \ell}(\bar{a}) \rightarrow \overline{W_{q}^{i, \ell}(a)}$ is proved as in [Cha01, Lemmas 2.3 and 2.4]. Hence it suffices to show that $\operatorname{dim}_{\mathbb{C}(q)} W_{q}^{i, \ell}(a)=\operatorname{dim}_{\mathbb{C}} W^{i, \ell}(\bar{a})$. This is proved in Cha01 for classical types, and deduced in general types as the special case of a single tensor factor of [Ked11, Corollary 5.1].

By the proposition, the pull-back $\varphi_{-\bar{a}}^{*}\left(\overline{W_{q}^{i, \ell}(a)}\right)$ is isomorphic to $W^{i, \ell}$, which has a graded $\mathfrak{g}[t]$-module structure. In this reason, $\varphi_{-\bar{a}}^{*}\left(\overline{W_{q}^{i, \ell}(a)}\right)$ is called the graded limit of $W_{q}^{i, \ell}(a)$.

We end this subsection with recalling a theorem in the case $\mathfrak{g}=\mathfrak{s l}_{2}$. In this case $I=\{1\}$ is a singleton set, and therefore we write $e, h, f$ for $e_{1}, h_{1}, f_{1}, W^{\ell}(c)$ for $W^{1, \ell}(c)$, and $W^{\ell}$ for $W^{1, \ell}$. Note that $W^{\ell}(c)$ is just the pull-back of the $(\ell+1)$-dimensional simple $\mathfrak{s l}_{2}$-module with respect to the evaluation map at $t=c$ :

$$
\mathfrak{s l}_{2}[t] \rightarrow \mathfrak{s l}_{2}: x \otimes f(t) \mapsto f(c) x \quad \text { for } x \in \mathfrak{s l}_{2}, f(t) \in \mathbb{C}[t] .
$$

The following is one of the main results in [FF02] (see also [CV15, Section 6]).

Theorem 2.9. Assume that $\mathfrak{g}=\mathfrak{s l}_{2}$, and define a power series $F(z) \in U\left(\mathfrak{s l}_{2}[t]\right)\left[\left[z^{-1}\right]\right]$ in an indeterminate $z$ by

$$
F(z)=\sum_{k=0}^{\infty}\left(f \otimes t^{k}\right) z^{-k-1}
$$


For a sequence $\ell_{1}, \ldots, \ell_{p}$ of positive integers, the fusion product $W^{\ell_{1}} * \cdots * W^{\ell_{p}}$ is isomorphic to the $\mathfrak{s l}_{2}[t]$-module generated by a vector $v$ with relations

$$
\begin{gathered}
(e \otimes \mathbb{C}[t]) v=0, \quad(h \otimes f(t)) v=\left(\ell_{1}+\cdots+\ell_{p}\right) f(0) v \text { for } f(t) \in \mathbb{C}[t], \\
\left(F(z)^{r}\right)_{s} v=0 \text { for } r>0, s<-\sum_{k=1}^{p} \min \left\{r, \ell_{k}\right\}
\end{gathered}
$$

where $\left(F(z)^{r}\right)_{s} \in U\left(\mathfrak{s l}_{2}[t]\right)$ denotes the coefficient of $z^{s}$ in the $r$-th power of $F(z)$.

\section{MAin TheOREM}

3.1. The statement of the main theorem. The following is the main theorem of this paper.

Theorem 3.1. Let $i_{1}, \ldots, i_{p} \in I, \ell_{1}, \ldots, \ell_{p} \in \mathbb{Z}_{>0}$, and $a_{1}, \ldots, a_{p} \in \mathcal{A}^{\times}$, and assume that the tensor product $W_{q}^{i_{1}, \ell_{1}}\left(a_{1}\right) \otimes \cdots \otimes W_{q}^{i_{p}, \ell_{p}}\left(a_{p}\right)$ is $\ell$-highest weight.

(i) If $a_{1}(1)=\cdots=a_{p}(1)=c \in \mathbb{C}^{\times}$, then the classical limit of the tensor product is isomorphic as a $\mathfrak{g}[t]$-module to the pull-back with respect to $\varphi_{c}$ of the fusion product of $W^{i_{k}, \ell_{k}}(1 \leq k \leq p)$, i.e.,

$$
\overline{W_{q}^{i_{1}, \ell_{1}}\left(a_{1}\right) \otimes \cdots \otimes W_{q}^{i_{p}, \ell_{p}}\left(a_{p}\right)} \cong \varphi_{c}^{*}\left(W^{i_{1}, \ell_{1}} * \cdots * W^{i_{p}, \ell_{p}}\right) .
$$

(ii) In the general case, define a finite subset $\mathcal{C}=\left\{a_{k}(1) \mid 1 \leq k \leq p\right\} \subseteq \mathbb{C}^{\times}$. Then the following isomorphism of $\mathfrak{g}[t]$-modules holds:

$$
\overline{W_{q}^{i_{1}, \ell_{1}}\left(a_{1}\right) \otimes \cdots \otimes W_{q}^{i_{p}, \ell_{p}}\left(a_{p}\right)} \cong \bigotimes_{c \in \mathcal{C}} \varphi_{c}^{*}\left(\underset{k ; a_{k}(1)=c}{*} W^{i_{k}, \ell_{k}}\right)
$$

where $* W^{i_{k}, \ell_{k}}$ denotes the fusion product of $W^{i_{k}, \ell_{k}}$ 's.

Remark 3.2. (a) As far as the author knows, no necessary and sufficient conditions are known for a tensor product of KR modules to be $\ell$-highest weight, but a sufficient condition has been obtained in Cha02.

(b) In the setting of the assertion (i), the graded $\mathfrak{g}[t]$-module

$$
\varphi_{-c}^{*}\left(\overline{W_{q}^{i_{1}, \ell_{1}}\left(a_{1}\right) \otimes \cdots \otimes W_{q}^{i_{p}, \ell_{p}}\left(a_{p}\right)}\right)
$$

is called the graded limit of $W_{q}^{i_{1}, \ell_{1}}\left(a_{1}\right) \otimes \cdots \otimes W_{q}^{i_{p}, \ell_{p}}\left(a_{p}\right)$. In this terminology, the assertion (i) claims that the graded limit of the tensor product is isomorphic to the fusion product of the graded limits.

(c) The $\mathfrak{g}[t]$-module $\varphi_{c}^{*}\left(W^{i_{1}, \ell_{1}} * \cdots * W^{i_{p}, \ell_{p}}\right)$ in the assertion (i) is uniquely lifted to an $\mathbf{L g}$-module via the Lie algebra homomorphism (2.11), and then the isomorphism in (i) becomes that of $\mathbf{L g}$-modules. The isomorphism in (ii) is also lifted to that of $\mathbf{L} \mathfrak{g}$-modules in the same way. 
3.2. Reduction to $\boldsymbol{U}_{\boldsymbol{q}}\left(\mathbf{L s t}_{\mathbf{2}}\right)$ case. Theorem 3.1 is proved by reducing it to the case of $U_{q}\left(\mathbf{L} \mathfrak{s l}_{2}\right)$, and for the reduction we need to prove another theorem which gives defining relations of the fusion product of $W^{i, \ell}$ 's (Theorem 3.3). In this subsection we will present the statement of Theorem 3.3, and then show that Theorem 3.1 is indeed reduced to the $U_{q}\left(\mathbf{L s l}_{2}\right)$ case (Proposition 3.5) via this theorem. The proofs of Theorem 3.3 and Proposition 3.5 will be given in the next two sections.

Define for each $i \in I$ a power series $F_{i}(z) \in U(\mathfrak{g}[t])\left[\left[z^{-1}\right]\right]$ by

$$
F_{i}(z)=\sum_{k=0}^{\infty}\left(f_{i} \otimes t^{k}\right) z^{-k-1}
$$

For any formal series $f(z)$ in $z$, denote by $f(z)_{s}(s \in \mathbb{Z})$ the coefficient of $z^{s}$. In Section 1, we will show the following theorem, which is a generalization of Theorem 2.9.

Theorem 3.3. Suppose that sequences $i_{1}, \ldots, i_{p}$ of elements of $I$ and $\ell_{1}, \ldots, \ell_{p}$ of positive integers are given. Set $\lambda=\sum_{k=1}^{p} \ell_{k} \varpi_{i_{k}}$, and define a subset

$$
S_{i}=\left\{1 \leq k \leq p \mid i_{k}=i\right\}
$$

for each $i \in I$. Then the fusion product $W^{i_{1}, \ell_{1}} * \cdots * W^{i_{p}, \ell_{p}}$ is isomorphic to the $\mathfrak{g}[t]$ module generated by a single vector $v$ with relations

$$
\begin{aligned}
\mathfrak{n}_{+}[t] v & =0, \quad(h \otimes f(t)) v=f(0)\langle h, \lambda\rangle v \text { for } h \in \mathfrak{h}, f(t) \in \mathbb{C}[t], \\
\left(F_{i}(z)^{r}\right)_{s} v & =0 \text { for } i \in I, r>0, s<-\sum_{k \in S_{i}} \min \left\{r, \ell_{k}\right\} .
\end{aligned}
$$

Remark 3.4. (a) For an $I$-tuple of partitions $\boldsymbol{\mu}=\left(\mu^{(1)}, \ldots, \mu^{(n)}\right)$ with $\mu^{(i)}=\left(\mu_{1}^{(i)} \geq\right.$ $\left.\ldots \geq \mu_{p_{i}}^{(i)}\right)$, denote by $W(\boldsymbol{\mu})$ the fusion product $*_{i, k} W^{i, \mu_{k}^{(i)}}$. Then Theorem $3.3 \mathrm{im}$ plies the following fact: if two $I$-tuples of partitions $\boldsymbol{\mu}=\left(\mu^{(1)}, \ldots, \mu^{(n)}\right)$ and $\boldsymbol{\nu}=$ $\left(\nu^{(1)}, \ldots, \nu^{(n)}\right)$ satisfy $\left|\mu^{(i)}\right|=\left|\nu^{(i)}\right|$ and $\mu^{(i)} \leq \nu^{(i)}$ (with respect to the dominance order) for all $i \in I$, then there exists a surjective $\mathfrak{g}[t]$-module homomorphism $W(\boldsymbol{\mu}) \rightarrow W(\boldsymbol{\nu})$. Indeed for every $i \in I$ and $r>0$, setting $k_{r}=\max \left\{k \mid \mu_{k}^{(i)}>r\right\}$, the assumption implies that

$$
\sum_{k \geq 1} \min \left\{r, \mu_{k}^{(i)}\right\}=r k_{r}+\sum_{k>k_{r}} \mu_{k}^{(i)} \geq r k_{r}+\sum_{k>k_{r}} \nu_{k}^{(i)} \geq \sum_{k \geq 1} \min \left\{r, \nu_{k}^{(i)}\right\},
$$

and hence a surjection $W(\boldsymbol{\mu}) \rightarrow W(\boldsymbol{\nu})$ exists by Theorem 3.3. This surjection can be viewed as an extension of the Schur positivity of KR-modules proved in FH14 to the current algebra setting (see also [Nao15]).

(b) In [CV15], the authors have introduced a collection of $\mathfrak{g}[t]$-modules $V(\boldsymbol{\xi})$ indexed by an $R^{+}$-tuple of partitions $\boldsymbol{\xi}=\left(\xi^{(\alpha)}\right)_{\alpha \in R^{+}}$satisfying $\left|\xi^{(\alpha)}\right|=\left\langle h_{\alpha}, \mu\right\rangle$ for some $\mu \in P^{+}$. In their terminology, Theorem 3.3 says that the module $W^{i_{1}, \ell_{1}} * \cdots * W^{i_{p}, \ell_{p}}$ is isomorphic to $V(\boldsymbol{\xi})$, where $\boldsymbol{\xi}=\left(\xi^{(\alpha)}\right)_{\alpha \in R^{+}}$is defined by

$$
\xi^{(\alpha)}= \begin{cases}\operatorname{part}\left\{\ell_{k} \mid k \in S_{i}\right\} & \text { if } \alpha=\alpha_{i}, \\ \left(1^{\left\langle h_{\alpha}, \lambda\right\rangle}\right) & \text { if } \alpha \text { is not simple. }\end{cases}
$$

Here part $T$ for a multiset of positive integers $T$ denotes the partition obtained by ordering the elements of $T$. 
We will prove the following proposition in Section 5. Though this is just a special case of Theorem 3.1 (i) for $\mathfrak{g}=\mathfrak{s l}_{2}$, we write the precise statement here for later reference (we write $W_{q}^{\ell}(a)$ for $W_{q}^{1, \ell}(a)$ and $W^{\ell}$ for $\left.W^{1, \ell}\right)$.

Proposition 3.5. Assume that $\mathfrak{g}=\mathfrak{s l}_{2}$. Let $\ell_{1}, \ldots, \ell_{p} \in \mathbb{Z}_{>0}$ and $a_{1}, \ldots, a_{p} \in \mathcal{A}^{\times}$be sequences such that $a_{1}(1)=\cdots=a_{p}(1)=c \in \mathbb{C}^{\times}$and $W_{q}^{\ell_{1}}\left(a_{1}\right) \otimes \cdots \otimes W_{q}^{\ell_{p}}\left(a_{p}\right)$ is $\ell$-highest weight. Then we have

$$
\overline{W_{q}^{\ell_{1}}\left(a_{1}\right) \otimes \cdots \otimes W_{q}^{\ell_{p}}\left(a_{p}\right)} \cong \varphi_{c}^{*}\left(W^{\ell_{1}} * \cdots * W^{\ell_{p}}\right)
$$

as $\mathfrak{s l}_{2}[t]$-modules.

Now we deduce Theorem 3.1 in full generality, assuming Theorem 3.3 and Proposition 3.5. First we show the assertion (i). Let $v_{k}(1 \leq k \leq p)$ denote an $\ell$-highest weight vector of $W_{q}^{i_{k}, \ell_{k}}\left(a_{k}\right)$. In this proof we use the following abbreviations:

$$
W_{q}=W_{q}^{i_{1}, \ell_{1}}\left(a_{1}\right) \otimes \cdots \otimes W_{q}^{i_{p}, \ell_{p}}\left(a_{p}\right), \quad v=v_{1} \otimes \cdots \otimes v_{p} \in W_{q} .
$$

Note that $v$ is an $\ell$-highest weight vector of $W_{q}$ by the assumption. Fix $i \in I$ for a moment, and let $k_{1}, \ldots, k_{p_{i}}$ be the subsequence of $1, \ldots, p$ such that

$$
\left\{k_{1}, \ldots, k_{p_{i}}\right\}=\left\{1 \leq k \leq p \mid i_{k}=i\right\} .
$$

By Lemma 2.4, the $U_{q, i}$-submodule $U_{q, i} v$ of $W_{q}$ is isomorphic, as a $U_{q}\left(\mathbf{L s l}_{2}\right)_{d_{i}}$-module, to

$$
W_{q}^{\ell_{k_{1}}}\left(a_{k_{1}}\right) \otimes \cdots \otimes W_{q}^{\ell_{k_{p_{i}}}}\left(a_{k_{p_{i}}}\right) .
$$

Obviously this is an $\ell$-highest weight $U_{q}\left(\mathbf{L s t}_{2}\right)_{d_{i}}$-module. Now we consider the classical limit $\overline{U_{q, i} v}=\mathbb{C} \otimes_{\mathcal{A}} U_{\mathcal{A}, i} v$, which is an $\mathbf{L s l}_{2, i}$-module. By Proposition 3.5, $\overline{U_{q, i} v}$ is isomorphic to $\varphi_{c}^{*}\left(W^{\ell_{k_{1}}} * \cdots * W^{\ell_{k_{p_{i}}}}\right)$ as an $\mathfrak{s l}_{2}[t]$-module, and hence it holds that

$$
\varphi_{-c}^{*} \overline{U_{q, i} v} \cong W^{\ell_{k_{1}}} * \cdots * W^{\ell_{p_{i}}}
$$

Then by Theorem 2.9, the vector $\bar{v}=1 \otimes v \in \varphi_{-c}^{*} \overline{U_{q, i} v}$ satisfies the relations

$$
\begin{aligned}
& \left(e_{i} \otimes \mathbb{C}[t]\right) \bar{v}=0, \quad\left(h_{i} \otimes f(t)\right) \bar{v}=f(0)\left\langle h_{i}, \lambda\right\rangle \bar{v} \text { for } f(t) \in \mathbb{C}[t], \\
& \left(F_{i}(z)^{r}\right)_{s} \bar{v}=0 \text { for } r>0, s<-\sum_{j=1}^{p_{i}} \min \left\{r, \ell_{k_{j}}\right\},
\end{aligned}
$$

where $\lambda=\sum_{k=1}^{p} \ell_{k} \varpi_{i_{k}}$. Moreover there is an $\mathfrak{s l}_{2, i}[t]$-module homomorphism $\overline{U_{q, i} v} \rightarrow \overline{W_{q}}$ since $U_{\mathcal{A}, i} v \subseteq U_{\mathcal{A}}(\mathbf{L} \mathfrak{g}) v$, which induces a homomorphism $\varphi_{-c}^{*} \overline{U_{q, i} v} \rightarrow \varphi_{-c}^{*} \overline{W_{q}}$. Hence $\bar{v} \in \varphi_{-c}^{*} \overline{W_{q}}$ also satisfies the relations (3.2). Now applying this argument to all $i \in I$, it follows from Theorem 3.3 that there exists a surjective $\mathfrak{g}[t]$-module homomorphism

$$
W^{i_{1}, \ell_{1}} * \cdots * W^{i_{p}, \ell_{p}} \rightarrow \varphi_{-c}^{*} \overline{W_{q}} .
$$

It follows from Proposition 2.8 that the dimensions of these modules are equal. Hence this is an isomorphism and the assertion (i) is proved.

Now the assertion (ii) of Theorem 3.1 is deduced from (i) as follows. For each $c \in \mathcal{C}$, set

$$
W_{q}^{(c)}=\bigotimes_{k ; a_{k}(1)=c} W_{q}^{i_{k}, \ell_{k}}\left(a_{k}\right)
$$


where the factors are ordered so that $W_{q}^{i_{r}, \ell_{r}}\left(a_{r}\right)$ is left to $W_{q}^{i_{s}, \ell_{s}}\left(a_{s}\right)$ if $r<s$. It follows from Lemma 2.3 (ii) that

$$
W_{q}^{i_{1}, \ell_{1}} \otimes \cdots \otimes W_{q}^{i_{p}, \ell_{p}} \cong \bigotimes_{c \in \mathcal{C}} W_{q}^{(c)}
$$

where the order of the right-hand side is arbitrary. Hence each $W_{q}^{(c)}$ is $\ell$-highest weight since so is $W_{q}^{i_{1}, \ell} \otimes \cdots \otimes W_{q}^{i_{p}, \ell_{p}}$. Let $v^{(c)}(c \in \mathcal{C})$ be an $\ell$-highest weight vector of $W_{q}^{(c)}$. Since $U_{\mathcal{A}}(\mathbf{L} \mathfrak{g})$ is a sub-coalgebra, we have

$$
U_{\mathcal{A}}(\mathbf{L} \mathfrak{g}) \bigotimes_{c \in \mathcal{C}} v^{(c)} \subseteq \bigotimes_{c \in \mathcal{C}} U_{\mathcal{A}}(\mathbf{L} \mathfrak{g}) v^{(c)}
$$

in $\bigotimes_{c} W_{q}^{(c)}$, and hence we obtain by (i) an $\mathbf{L} \mathfrak{g}$-module homomorphism

$$
\overline{\bigotimes_{c \in \mathcal{C}} W_{q}^{(c)}} \rightarrow \bigotimes_{c \in \mathcal{C}} \overline{W_{q}^{(c)}} \cong \bigotimes_{c \in \mathcal{C}} \varphi_{c}^{*}\left(\underset{k ; a_{k}(1)=c}{*} W^{i_{k}, \ell_{k}}\right)
$$

By [FL99, Proposition 1.4] the module $\bigotimes_{c} \varphi_{c}^{*}\left(* W^{i_{k}, \ell_{k}}\right)$ is generated by the image of $\overline{\bigotimes_{c} v^{(c)}}$, and hence the homomorphism is surjective. Then the comparison of the dimensions shows that this is an isomorphism, and (ii) is proved.

\section{Proof of Theorem 3.3}

4.1. Reduction to a study of a quotient space of $\boldsymbol{U}\left(\mathbf{L} \mathfrak{n}_{-}\right)$. This section is devoted to the proof of Theorem 3.3. Fix sequences $i_{1}, \ldots, i_{p}$ of elements of $I$ and $\ell_{1}, \ldots, \ell_{p}$ of positive integers. As in the theorem, set $\lambda=\sum_{k} \ell_{k} \varpi_{i_{k}}$ and $S_{i}=\left\{1 \leq k \leq p \mid i_{k}=i\right\}$.

Write $W=W^{i_{1}, \ell_{1}} * \cdots * W^{i_{p}, \ell_{p}}$, and let $M$ denote the $\mathfrak{g}[t]$-module generated by a vector $v$ with relations (3.1). We begin with the following lemma.

Lemma 4.1. There exists a surjective $\mathfrak{g}[t]$-module homomorphism from $M$ to $W$.

Proof. Let $v_{k}(1 \leq k \leq p)$ denote the generator of $W^{i_{k}, \ell_{k}}$. For $i \in I$, it is clear from the defining relations that the $\mathfrak{s l}_{2, i}[t]$-submodule $U\left(\mathfrak{s l}_{2, i}[t]\right) v_{k} \subseteq W^{i_{k}, \ell_{k}}$ is isomorphic to $W^{\ell_{k}}$ if $k \in S_{i}$, and a trivial module otherwise. Hence, letting $c_{1}, \ldots, c_{p}$ be pairwise distinct complex numbers, there is an injective $\mathfrak{s l}_{2, i}[t]$-module homomorphism

$$
\bigotimes_{k \in S_{i}} \varphi_{c_{k}}^{*} W^{\ell_{k}} \hookrightarrow \bigotimes_{k=1}^{p} \varphi_{c_{k}}^{*} W^{i, \ell_{k}} .
$$

By the definition of the fusion product, this induces, for every $i \in I$, an $\mathfrak{s l}_{2, i}[t]$-module homomorphism from the fusion product $*_{k \in S_{i}} W^{\ell_{k}}$ to $W$. Then it follows from Theorem 2.9 that the generator of $W$ satisfies the relations (3.1). Hence there is a $\mathfrak{g}[t]$-module homomorphism from $M$ to $W$, which is obviously surjective. The lemma is proved.

Since both $M$ and $W$ are finite-dimensional $\mathfrak{g}$-modules, Lemma 4.1 implies that, for the proof of Theorem 3.3, it suffices to show the inequalities

$$
[M: V(\mu)] \leq[W: V(\mu)]
$$


of multiplicities as $\mathfrak{g}$-modules for all $\mu \in P^{+}$. To show this, we will give an upper bound of $[M: V(\mu)]$ below.

To begin with, note that the following equality follows from the finite-dimensional representation theory of $\mathfrak{g}$ :

$$
[M: V(\mu)]=\operatorname{dim}\left(M / \mathfrak{n}_{-} M\right)_{\mu}
$$

Here $M / \mathfrak{n}_{-} M$ is $P$-graded by the action of $\mathfrak{h}$.

To shorten the notation we write $U^{-}$for $U\left(\mathfrak{n}_{-}[t]\right)$ from now on. Let $\mathcal{I}$ be the left $U^{-}$-ideal generated by the vectors

$$
\left\{\left(F_{i}(z)^{r}\right)_{s} \mid i \in I, r>0, s<-\sum_{k \in S_{i}} \min \left\{r, \ell_{k}\right\}\right\}
$$

The $\mathfrak{n}_{-}[t]$-module $U^{-} / \mathcal{I}$ is naturally graded by $-Q^{+}$, and obviously there exists a surjective $\mathfrak{n}_{-}[t]$-module homomorphism from $U^{-} / \mathcal{I}$ to $M$, which maps $\left(U^{-} / \mathcal{I}\right)_{-\gamma}$ for $\gamma \in Q^{+}$onto $M_{\lambda-\gamma}$. Then this homomorphism yields the following surjective linear map

$$
\left(U^{-} /\left(\mathfrak{n}_{-} U^{-}+\mathcal{I}\right)\right)_{-\gamma} \rightarrow\left(M / \mathfrak{n}_{-} M\right)_{\lambda-\gamma},
$$

which implies the inequality

$$
\operatorname{dim}\left(U^{-} /\left(\mathfrak{n}_{-} U^{-}+\mathcal{I}\right)\right)_{-\gamma} \geq[M: V(\lambda-\gamma)]
$$

by (4.1).

Next we will define a quotient space of $U\left(\mathbf{L} \mathfrak{n}_{-}\right)$which is linearly isomorphic to $U^{-} /\left(\mathfrak{n}_{-} U^{-}+\mathcal{I}\right)$. In the sequel we write $U_{\mathbf{L}}^{-}=U\left(\mathbf{L n}_{-}\right)$for ease of notation. Fix a sufficiently large positive integer $N$, and let $\widetilde{F}_{i}(z) \in U_{\mathbf{L}}^{-}((z))$ be the formal Laurent series defined by

$$
\widetilde{F}_{i}(z)=\sum_{k=-\infty}^{N}\left(f_{i} \otimes t^{k}\right) z^{-k-1} .
$$

Denote by $\mathcal{J}$ the left $U_{\mathbf{L}}^{-}$-ideal generated by the vectors

$$
\left\{f_{i} \otimes t^{k} \mid i \in I, k>N\right\} \cup\left\{\left(\widetilde{F}_{i}(z)^{r}\right)_{s} \mid i \in I, r>0, s<-\sum_{k \in S_{i}} \min \left\{r, \ell_{k}\right\}\right\} .
$$

The definition of $\mathcal{J}$ does not depend on the choice of $N$, since

$$
\widetilde{F}_{i}(z)_{-k-1}=f_{i} \otimes t^{k} \in \mathcal{J} \text { for } \# S_{i} \leq k \leq N .
$$

Lemma 4.2. (i) The left ideal $U_{\mathbf{L}}^{-} \mathcal{I}$ coincides with $\mathcal{J}$.

(ii) There exists a linear isomorphism

$$
U_{\mathbf{L}}^{-} /\left(\mathfrak{n}_{-}\left[t^{-1}\right] U_{\mathbf{L}}^{-}+\mathcal{J}\right) \stackrel{\sim}{\rightarrow} U^{-} /\left(\mathfrak{n}_{-} U^{-}+\mathcal{I}\right)
$$

preserving their $-Q^{+}$-gradings. 
Proof. (i) We show $U_{\mathbf{L}}^{-} \mathcal{I} \subseteq \mathcal{J}$ (the other containment is similarly proved). For $i \in I$, set

$$
Z_{i}=\left\{(r, s) \mid r>0, s<-\sum_{k \in S_{i}} \min \left\{r, \ell_{k}\right\}\right\} \subseteq \mathbb{Z}_{>0} \times \mathbb{Z},
$$

and $F_{i}^{\prime}(z)=\sum_{k=0}^{N}\left(f_{i} \otimes t^{k}\right) z^{-k-1}$. Since $f_{i} \otimes t^{k} \in \mathcal{J}$ for $k>N$, it suffices to show that $\left(F_{i}^{\prime}(z)^{r}\right)_{s} \in \mathcal{J}$ if $(r, s) \in Z_{i}$. We show by the induction on $k$ that

$$
\left(F_{i}^{\prime}(z)^{k} \widetilde{F}_{i}(z)^{r-k}\right)_{s} \in \mathcal{J} \text { if }(r, s) \in Z_{i},
$$

which with $k=r$ completes the proof. For $k=0$ there is nothing to prove. When $k>0$, we have

$$
\begin{aligned}
& \left(F_{i}^{\prime}(z)^{k} \widetilde{F}_{i}(z)^{r-k}\right)_{s} \\
& \quad=\left(F_{i}^{\prime}(z)^{k-1} \widetilde{F}_{i}(z)^{r-k+1}-\sum_{a<0} z^{-a-1}\left(f_{i} \otimes t^{a}\right) F_{i}^{\prime}(z)^{k-1} \widetilde{F}_{i}(z)^{r-k}\right)_{s} \\
& \quad=\left(F_{i}^{\prime}(z)^{k-1} \widetilde{F}_{i}(z)^{r-k+1}\right)_{s}-\sum_{a<0} f_{i} \otimes t^{a}\left(F_{i}^{\prime}(z)^{k-1} \widetilde{F}_{i}(z)^{r-k}\right)_{s+a+1}
\end{aligned}
$$

Since $(r, s) \in Z_{i}$ implies $(r-1, s+a+1) \in Z_{i}$ for all $a<0$, the right-hand side belongs to $\mathcal{J}$ by the induction hypothesis. The proof is complete.

(ii) By the Poincaré-Birkhoff-Witt theorem, we have

$$
U_{\mathbf{L}}^{-}=\left(t^{-1} \mathfrak{n}_{-}\left[t^{-1}\right]\right) U_{\mathbf{L}}^{-} \oplus U^{-} .
$$

Consider the composition

$$
U_{\mathbf{L}}^{-} \rightarrow U^{-} \rightarrow U^{-} /\left(\mathfrak{n}_{-} U^{-}+\mathcal{I}\right),
$$

where the first map is the projection with respect to the above decomposition, and the second is the canonical one. Obviously the kernel of this linear map is

$$
\left(t^{-1} \mathfrak{n}_{-}\left[t^{-1}\right]\right) U_{\mathbf{L}}^{-}+\mathfrak{n}_{-} U^{-}+\mathcal{I}
$$

which we denote by $\mathcal{K}$. It suffices to show that

$$
\mathcal{K}=\mathfrak{n}_{-}\left[t^{-1}\right] U_{\mathbf{L}}^{-}+\mathcal{J},
$$

and the containment $\subseteq$ is clear from (i). We show the other containment. Since $\operatorname{ad}\left(\mathfrak{n}_{-}\right)\left(t^{-1} \mathfrak{n}_{-}\left[t^{-1}\right]\right) \subseteq t^{-1} \mathfrak{n}_{-}\left[t^{-1}\right]$, we have

$$
\mathfrak{n}_{-} U_{\mathbf{L}}^{-}=\mathfrak{n}_{-}\left(\left(t^{-1} \mathfrak{n}_{-}\left[t^{-1}\right]\right) U_{\mathbf{L}}^{-} \oplus U^{-}\right) \subseteq\left(t^{-} \mathfrak{n}_{-}\left[t^{-1}\right]\right) U_{\mathbf{L}}^{-}+\mathfrak{n}_{-} U^{-} \subseteq \mathcal{K},
$$

and hence $\mathfrak{n}_{-}\left[t^{-1}\right] U_{\mathbf{L}}^{-} \subseteq \mathcal{K}$ holds. On the other hand, it also follows from (i) that

$$
\mathcal{J}=U_{\mathbf{L}}^{-} \mathcal{I}=\left(\left(t^{-1} \mathfrak{n}_{-}\left[t^{-1}\right]\right) U_{\mathbf{L}}^{-} \oplus U^{-}\right) \mathcal{I} \subseteq\left(t^{-1} \mathfrak{n}_{-}\left[t^{-1}\right]\right) U_{\mathbf{L}}^{-}+\mathcal{I} \subseteq \mathcal{K}
$$

Therefore $\mathcal{K} \supseteq \mathfrak{n}_{-}\left[t^{-1}\right] U_{\mathbf{L}}^{-}+\mathcal{J}$ follows, as required.

In conclusion, the proof of Theorem 3.3 is now reduced to the following proposition by (4.2) and Lemma 4.2 (ii), which will be proved in Subsection 4.3. 
Proposition 4.3. For each $\gamma \in Q^{+}$such that $\lambda-\gamma \in P^{+}$, the inequality

$$
\operatorname{dim}\left(U_{\mathbf{L}}^{-} /\left(\mathfrak{n}_{-}\left[t^{-1}\right] U_{\mathbf{L}}^{-}+\mathcal{J}\right)\right)_{-\gamma} \leq[W: V(\lambda-\gamma)]
$$

holds.

4.2. Functional realization of the dual space of $\boldsymbol{U}_{\mathbf{L}}^{-}$. Proposition 4.3 is proved using the functional realization of the dual space of $U_{\mathbf{L}}^{-}$introduced in FS94 (see also [FKL ${ }^{+}$02], [AKS06], AK07]). Here we give a detailed exposition of the realization.

For $i, j \in I$, write $\widehat{c_{i j}}=\max \left\{1-c_{i j}, 1\right\}$. Let $\gamma=\sum_{i \in I} m_{i} \alpha_{i} \in Q^{+}$, and define $\mathcal{U}_{\gamma}$ by the subspace of the space of rational functions in the variables

$$
\boldsymbol{x}_{\gamma}=\left\{x_{r}^{(i)} \mid i \in I, 1 \leq r \leq m_{i}\right\},
$$

consisting of the functions $g\left(\boldsymbol{x}_{\gamma}\right)$ of the form

$$
g\left(\boldsymbol{x}_{\gamma}\right)=\frac{g^{\prime}\left(\boldsymbol{x}_{\gamma}\right)}{\prod_{i<j} \prod_{\substack{1 \leq r \leq m_{i} \\ 1 \leq s \leq m_{j}}}\left(x_{r}^{(i)}-x_{s}^{(j)}\right)},
$$

where $g^{\prime}\left(\boldsymbol{x}_{\gamma}\right) \in \mathbb{C}\left[\left(x_{r}^{(i)}\right)^{ \pm 1} \mid i, r\right]$ is a Laurent polynomial, symmetric under the exchange of variables $x_{r}^{(i)} \leftrightarrow x_{s}^{(i)}$ with the same superscript, and vanishes provided $x_{1}^{(i)}=x_{2}^{(i)}=$ $\cdots=x_{\widehat{c}_{i j}}^{(i)}=x_{1}^{(j)}$ for $i \neq j$. We will write $\boldsymbol{x}_{\gamma}$ simply as $\boldsymbol{x}$ when $\gamma$ is obvious from the context. Let $\mathcal{U}=\bigoplus_{\gamma \in Q^{+}} \mathcal{U}_{\gamma}$.

For $\gamma \in Q^{+}, i \in I$ and $k \in \mathbb{Z}$, define a map $R_{i, k}: \mathcal{U}_{\gamma} \rightarrow \mathcal{U}_{\gamma-\alpha_{i}}$ by

$$
R_{i, k}(g(\boldsymbol{x}))=\operatorname{Res}_{x_{1}^{(i)}}\left(x_{1}^{(i)}\right)^{k} g(\boldsymbol{x}),
$$

and extend it on $\mathcal{U}$ linearly. Here the residue $\operatorname{Res}_{x} g(\boldsymbol{x})$ for a variable $x$ is defined as follows: first regard $g(\boldsymbol{x})$ as a formal Laurent series in $x$ by expanding all $\left(x-x_{r}^{(i)}\right)^{-1}$ in positive power of $x / x_{r}^{(i)}$, and then take the coefficient of $x^{-1}$.

Remark 4.4. Precisely to say, in order to define $R_{i, k}(g(\boldsymbol{x}))$ as a function in $\mathcal{U}_{\gamma-\alpha_{i}}$, we need to reindex the variables $\left\{x_{2}^{(i)}, \ldots, x_{m_{i}}^{(i)}\right\}$ to $\left\{x_{1}^{(i)}, \ldots, x_{m_{i}-1}^{(i)}\right\}$ (any reindex produces a same function because of the symmetry). In the sequel, we always assume such an obvious reindexing is done, if necessary, without any mention.

If a rational function $g(x, y)$ in two variables $x$ and $y$ has no poles except at $x=0$, $y=0$ and $x=y$, it follows from the Cauthy's residue theorem that

$$
\left(\operatorname{Res}_{y} \operatorname{Res}_{x}-\operatorname{Res}_{x} \operatorname{Res}_{y}\right) g(x, y)=-\operatorname{Res}_{y} \operatorname{Res}_{x=y} g(x, y) \text {. }
$$

From this, a useful formula is deduced as follows. Suppose that $h \in \mathbb{Z}_{>0}, i_{1}, \ldots, i_{h} \in I$ and $k_{1}, \ldots, k_{h} \in \mathbb{Z}$ are given. Set $\beta=\alpha_{i_{1}}+\cdots+\alpha_{i_{h}}$, and rename the variables $\boldsymbol{x}_{\beta}$ into $\left\{x_{1}, \ldots, x_{h}\right\}$ by

$$
x_{r}=x_{\#\left\{s \leq r \mid i_{s}=i_{r}\right\}}^{\left(i_{r}\right)} \text { for } 1 \leq r \leq h .
$$

Then in view of (4.3), we have

$$
\begin{aligned}
& {\left[R_{i_{h}, k_{h}},\left[R_{i_{h-1}, k_{h-1}}, \ldots,\left[R_{i_{2}, k_{2}}, R_{i_{1}, k_{1}}\right] \ldots\right]\right] g(\boldsymbol{x})} \\
& \quad=(-1)^{h-1} \operatorname{Res}_{x_{h}}\left(x_{h}^{k_{h}} \operatorname{Res}_{x_{h-1}=x_{h}}\left(x_{h-1}^{k_{h-1}} \cdots \operatorname{Res}_{x_{1}=x_{2}}\left(x_{1}^{k_{1}} g(\boldsymbol{x})\right) \cdots\right)\right)
\end{aligned}
$$


for $g(\boldsymbol{x}) \in \mathcal{U}$. Moreover for each $2 \leq r \leq h-1$, it can be proved that the function

$$
x_{r}^{k_{r}} \operatorname{Res}_{x_{r-1}=x_{r}}\left(x_{r-1}^{k_{r-1}} \cdots \operatorname{Res}_{x_{1}=x_{2}}\left(x_{1}^{k_{1}} g(\boldsymbol{x})\right) \cdots\right)
$$

has at most a simple pole at $x_{r}=x_{r+1}$ (we will give a proof of this fact in Appendix A.2 since we have not found one in the literature). Hence the above formula is rewritten in the following simpler form:

$$
\begin{aligned}
& {\left[R_{i_{h}, k_{h}},\left[R_{i_{h-1}, k_{h-1}}, \ldots,\left[R_{i_{2}, k_{2}}, R_{i_{1}, k_{1}}\right] \ldots\right]\right] g(\boldsymbol{x})} \\
& \quad=\operatorname{Res}_{x_{h}}\left\{\left.\prod_{r=2}^{h}\left(x_{r}-x_{r-1}\right) g(\boldsymbol{x})\right|_{x_{1}=x_{2}=\cdots=x_{h}} \cdot\left(x_{h}\right)^{k_{1}+\cdots+k_{h}}\right\} .
\end{aligned}
$$

In the sequel, we write $f_{\alpha, k}=f_{\alpha} \otimes t^{k}$ and $f_{i, k}=f_{i} \otimes t^{k}$ to shorten the notation. Define a bilinear map $\langle\rangle:, U_{\mathbf{L}}^{-} \times \mathcal{U} \rightarrow \mathcal{U}$ by

$$
\left\langle f_{i_{1}, k_{1}} f_{i_{2}, k_{2}} \cdots f_{i_{N}, k_{N}}, g(\boldsymbol{x})\right\rangle=R_{i_{1}, k_{1}} R_{i_{2}, k_{2}} \cdots R_{i_{N}, k_{N}} g(\boldsymbol{x}),
$$

where $i_{1}, \ldots, i_{N} \in I$ and $k_{1}, \ldots, k_{N} \in \mathbb{Z}$.

Proposition 4.5. The bilinear map $\langle$,$\rangle is well-defined.$

Proof. Since $U_{\mathbf{L}}^{-}$is generated by $\left\{f_{i, k} \mid i \in I, k \in \mathbb{Z}\right\}$ with relations

$$
\begin{aligned}
{\left[f_{i, k_{\widehat{c}_{i j}}},\left[f_{i, k_{\widehat{c}_{i j}-1}}, \ldots,\left[f_{i, k_{1}}, f_{j, l}\right] \ldots\right]\right] } & =0 \quad\left(i, j \in I, k_{r}, l \in \mathbb{Z}\right), \text { and } \\
{\left[f_{i_{h}, k_{h}},\left[f_{i_{h-1}, k_{h-1}}, \ldots,\left[f_{i_{2}, k_{2}}, f_{i_{1}, k_{1}}\right] \ldots\right]\right] } & =\left[f_{i_{h}, l_{h}},\left[f_{i_{h-1}, l_{h-1}}, \ldots,\left[f_{i_{2}, l_{2}}, f_{i_{1}, l_{1}}\right] \ldots\right]\right] \\
\left(h \in \mathbb{Z}_{>0}, i_{r} \in I, k_{r}, l_{r}\right. & \left.\in \mathbb{Z} \text { such that } k_{1}+\cdots+k_{h}=l_{1}+\cdots+l_{h}\right),
\end{aligned}
$$

it suffices to show for arbitrary $g \in \mathcal{U}$ that

$$
\begin{aligned}
{\left[R_{i, k_{\widehat{c}_{i j}}},\left[R_{i, k_{\widehat{c}_{i j}-1}}, \ldots,\left[R_{i, k_{1}}, R_{j, l}\right] \ldots\right]\right] g } & =0, \text { and } \\
{\left[R_{i_{h}, k_{h}},\left[R_{i_{h-1}, k_{h-1}}, \ldots,\left[R_{i_{2}, k_{2}}, R_{i_{1}, k_{1}}\right] \ldots\right]\right] g } & =\left[R_{i_{h}, l_{h}},\left[R_{i_{h-1}, l_{h-1}}, \ldots,\left[R_{i_{2}, l_{2}}, R_{i_{1}, l_{1}}\right] \ldots\right]\right] g .
\end{aligned}
$$

These are easily deduced from (4.4) and the definition of $\mathcal{U}$, and hence the assertion is proved.

For $\gamma \in Q^{+},\langle$,$\rangle defines a pairing \left(U_{\mathbf{L}}^{-}\right)_{-\gamma} \times \mathcal{U}_{\gamma} \rightarrow \mathbb{C}$. Note that $\left(U_{\mathbf{L}}^{-}\right)_{-\gamma}$ is naturally $\mathbb{Z}$-graded by the degree of polynomials, and so is $\mathcal{U}_{\gamma}$ by the total degree. It is easily checked that, for $F \in\left(U_{\mathbf{L}}^{-}\right)_{-\gamma}^{k}$ and $g \in \mathcal{U}_{\gamma}^{l},\langle F, g\rangle=0$ unless $k+l=-\operatorname{ht}(\gamma)$.

Let $\overline{\mathcal{U}}_{\gamma}$ be the subspace of $\mathcal{U}_{\gamma}$ consisting of $g(\boldsymbol{x})$ such that

$$
\left\langle f_{i, k}\left(U_{\mathbf{L}}^{-}\right)_{-\gamma+\alpha_{i}}, g(\boldsymbol{x})\right\rangle=0 \text { for all } i \in I, k \in \mathbb{Z}_{\leq 0} .
$$

By the definition of the pairing, this is equivalent to that, if we regard $g(\boldsymbol{x})$ as a formal Laurent series in $\left(x_{1}^{(i)}\right)^{-1}$ by expanding all $\left(x_{1}^{(i)}-x_{s}^{(j)}\right)^{-1}$ in positive power of $x_{s}^{(j)} / x_{1}^{(i)}$, then the coefficient of $\left(x_{1}^{(i)}\right)^{-k-1}$ is 0 for all $k \in \mathbb{Z}_{\leq 0}$. Hence the following lemma holds.

Lemma 4.6. We have

$$
\overline{\mathcal{U}}_{\gamma}=\left\{g(\boldsymbol{x}) \in \mathcal{U}_{\gamma} \mid \operatorname{deg}_{x_{1}^{(i)}} g(\boldsymbol{x}) \leq-2 \text { for all } i \in I\right\}
$$


where $\operatorname{deg}_{x_{1}^{(i)}}$ is defined by setting $\operatorname{deg}_{x_{1}^{(i)}} x_{r}^{(j)}=\delta_{(i, 1),(j, r)}$ and

$$
\operatorname{deg}_{x_{1}^{(i)}}\left(x_{r}^{(j)}-x_{s}^{\left(j^{\prime}\right)}\right)^{-1}= \begin{cases}-1 & \text { if }(j, r)=(i, 1) \text { or }\left(j^{\prime}, s\right)=(i, 1), \\ 0 & \text { otherwise. }\end{cases}
$$

Set $\bar{U}_{\mathbf{L}}^{-}=U_{\mathbf{L}}^{-} /\left(\mathfrak{n}_{-}\left[t^{-1}\right] U_{\mathbf{L}}^{-}\right)$. Obviously $\langle$,$\rangle induces a pairing between \left(\bar{U}_{\mathbf{L}}^{-}\right)_{-\gamma}$ and $\overline{\mathcal{U}}_{\gamma}$, and moreover the following proposition holds.

Proposition 4.7. The pairing induces a linear isomorphism $\overline{\mathcal{U}}_{\gamma} \stackrel{\sim}{\rightarrow}\left(\bar{U}_{\mathbf{L}}^{-}\right)_{-\gamma}^{\vee}$, where $\left(\bar{U}_{\mathbf{L}}^{-}\right)_{-\gamma}^{\vee}$ denotes the restricted dual $\bigoplus_{k \in \mathbb{Z}}\left(\left(\bar{U}_{\mathbf{L}}^{-}\right)_{-\gamma}^{k}\right)^{*}$.

This proposition is proved in Appendix A.3.

4.3. Proof of Proposition 4.3. Now we return to the setting of Subsection 4.1. Through the isomorphism $\overline{\mathcal{U}}_{\gamma} \stackrel{\sim}{\rightarrow}\left(\bar{U}_{\mathbf{L}}^{-}\right)_{-\gamma}^{\vee}$, the dual space of $\left(U_{\mathbf{L}}^{-} /\left(\mathfrak{n}_{-}\left[t^{-1}\right] U_{\mathbf{L}}^{-}+\mathcal{J}\right)\right)_{-\gamma}$ is regarded as a subspace of $\overline{\mathcal{U}}_{\gamma}$, consisting of the functions $g(\boldsymbol{x})$ satisfying $\langle\mathcal{J}, g(\boldsymbol{x})\rangle=0$. Set $\widehat{F}_{i}(z)=\sum_{k=-\infty}^{\infty} f_{i, k} z^{-k-1}$. Then for $r>0$, we have

$$
\left\langle\widehat{F}_{i}(z)^{r}, g(\boldsymbol{x})\right\rangle=\left.g(\boldsymbol{x})\right|_{x_{1}^{(i)}=\cdots=x_{r}^{(i)}=z} .
$$

(Here the coefficients of $\widehat{F}_{i}(z)^{r}$ belong to a completion of $U_{\mathbf{L}}^{-}$, but the pairing is still well-defined.) Let $\mathcal{V}_{\gamma}$ denote the subspace of $\overline{\mathcal{U}}_{\gamma}$ consisting of the functions $g(\boldsymbol{x})$ such that, for every $i \in I$ and $r>0$, the order of the pole of $\left.g(\boldsymbol{x})\right|_{x_{1}^{(i)}=\cdots=x_{r}^{(i)}=z}$ at $z=0$ is at most $\sum_{k \in S_{i}} \min \left\{r, \ell_{k}\right\}$. Then it follows from the definition of $\mathcal{J}$ that

$$
\left(U_{\mathbf{L}}^{-} /\left(\mathfrak{n}_{-}\left[t^{-1}\right] U_{\mathbf{L}}^{-}+\mathcal{J}\right)\right)_{-\gamma}^{*} \cong \mathcal{V}_{\gamma}
$$

Moreover, an upper bound of the dimension of $\mathcal{V}_{\gamma}$ is given as follows.

Lemma 4.8. Let $\lambda=\sum_{k} \ell_{k} \varpi_{i_{k}}$. For $\gamma \in Q^{+}$such that $\lambda-\gamma \in P^{+}$, we have

$$
\operatorname{dim} \mathcal{V}_{\gamma} \leq \sum_{\left\{m_{a}^{(i)}\right\}} \prod_{\substack{i \in I \\
a>0}}\left(\begin{array}{c}
p_{a}^{(i)}+m_{a}^{(i)} \\
m_{a}^{(i)}
\end{array}\right)
$$

where

$$
p_{a}^{(i)}=\sum_{k \in S_{i}} \min \left\{a, \ell_{k}\right\}+\sum_{\substack{j \in I \backslash\{i\} \\ b>0}} \min \left\{\left|c_{j i}\right| a,\left|c_{i j}\right| b\right\} m_{b}^{(j)}-2 \sum_{b>0} \min \{a, b\} m_{b}^{(i)},
$$

and the sum $\sum_{\left\{m_{a}^{(i)}\right\}}$ is taken over $\left\{m_{a}^{(i)} \in \mathbb{Z}_{\geq 0} \mid i \in I, a>0\right\}$ satisfying $p_{a}^{(i)} \geq 0$ for all $i, a$, and $\gamma=\sum_{i, a} a m_{a}^{(i)} \varpi_{i}$.

This lemma is proved by a similar argument given in AK07. For the reader's convenience, we reproduce it in Appendix A.4. 
By combining several results in $\mathrm{HKO}^{+} 99$, Nak03, Her06, DFK08] (see [DFK08, Subsections 2.3 and 2.4] for the details), it is shown that the right-hand side of (4.6) is equal to the $U_{q}(\mathfrak{g})$-module multiplicity

$$
\left[W_{q}^{i_{1}, \ell_{1}}\left(a_{1}\right) \otimes \cdots \otimes W_{q}^{i_{p}, \ell_{p}}\left(a_{p}\right): V_{q}(\lambda-\gamma)\right] .
$$

Since this multiplicity coincides with the $\mathfrak{g}$-module multiplicity $[W: V(\lambda-\gamma)]$, Proposition 4.3 now follows from Lemma 4.8 and (4.5). This completes the proof of Theorem 3.3, as explained above.

\section{Proof of Proposition 3.5}

5.1. Quantum loop algebra of type $\boldsymbol{A}_{1}^{(1)}$. Throughout this section we focus on the case $\mathfrak{g}=\mathfrak{s l}_{2}$ only. In what follows, we fix a positive integer $d$, and denote by $U_{q}\left(\mathbf{L s l}_{2}\right)$ the quantum loop algebra associated with $\widehat{C}=\left(\begin{array}{cc}2 & -2 \\ -2 & 2\end{array}\right)$ and $\widehat{D}=\left(\begin{array}{ll}d & 0 \\ 0 & d\end{array}\right)$ (which implies $\left.q_{0}=q_{1}=q^{d}\right)$. Let $\tilde{q}=q^{d}$.

Here we collect several results concerning finite-dimensional modules over $U_{q}\left(\mathbf{L s t}_{2}\right)$ (recall that we write $W_{q}^{\ell}(a)$ for $W_{q}^{1, \ell}(a)$ ).

Lemma 5.1. (i) $W_{q}^{\ell}(a)$ is simple as a $U_{q}\left(\mathfrak{s l}_{2}\right)$-module and of dimension $\ell+1$.

(ii) The $U_{q}\left(\mathbf{L s l}_{2}\right)$-submodule of $W_{q}^{\ell_{1}}\left(a_{1}\right) \otimes W_{q}^{\ell_{2}}\left(a_{2}\right)$ generated by the tensor product of $\ell$-highest weight vectors is proper and simple if

$$
a_{2} / a_{1} \in\left\{\tilde{q}^{2 k} \mid \max \left\{\ell_{1}-\ell_{2}, 0\right\}<k \leq \ell_{1}\right\} .
$$

(iii) $W_{q}^{\ell_{1}}\left(a_{1}\right) \otimes W_{q}^{\ell_{2}}\left(a_{2}\right)$ is simple if

$$
a_{2} / a_{1} \notin\left\{\tilde{q}^{2 k} \mid-\ell_{2} \leq k<\min \left\{\ell_{1}-\ell_{2}, 0\right\}, \max \left\{\ell_{1}-\ell_{2}, 0\right\}<k \leq \ell_{1}\right\} .
$$

In particular if this condition holds, then it follows from Lemma 2.3 (i) that

$$
W_{q}^{\ell_{1}}\left(a_{1}\right) \otimes W_{q}^{\ell_{2}}\left(a_{2}\right) \cong W_{q}^{\ell_{2}}\left(a_{2}\right) \otimes W_{q}^{\ell_{1}}\left(a_{1}\right) .
$$

(iv) If $\ell_{1}+\cdots+\ell_{p}=\ell$, then the $U_{q}\left(\mathbf{L s l}_{2}\right)$-submodule of

$$
W_{q}^{\ell_{1}}(a) \otimes W_{q}^{\ell_{2}}\left(\tilde{q}^{2 \ell_{1}} a\right) \otimes \cdots \otimes W_{q}^{\ell_{p}}\left(\tilde{q}^{2\left(\ell_{1}+\cdots+\ell_{p-1}\right)} a\right)
$$

generated by the tensor product of $\ell$-highest weight vectors is isomorphic to $W_{q}^{\ell}(a)$.

Proof. The proofs of (i)-(iii) are found in CP91 (note that the coproduct in the paper is different from ours). Then (iv) follows from (ii).

The modules $W_{q}^{1}(a)$ are called fundamental modules. The following lemma is deduced from (the proof of) [CP91, Lemma 4.10] (see also [Cha02, Theorem 2.6]).

Lemma 5.2. The tensor product of fundamental modules $W_{q}^{1}\left(a_{1}\right) \otimes \cdots \otimes W_{q}^{1}\left(a_{p}\right)$ is -highest weight if

$$
a_{s} / a_{r} \neq \tilde{q}^{2} \text { for all } 1 \leq r<s \leq p \text {. }
$$


5.2. Realization of fusion products. Write $e, f, h$ for $e_{1}, f_{1}, h_{1} \in \mathfrak{s l}_{2}$, and $W^{\ell}$ for the $\mathfrak{s l}_{2}[t]$-module $W^{1, \ell}$. Here we will recall a certain realization of a fusion product of $W^{\ell}$ 's, and for that we prepare some notation.

Let $\widehat{\mathfrak{s l}_{2}}=\mathfrak{s l}_{2} \otimes \mathbb{C}\left[t, t^{-1}\right] \oplus \mathbb{C} K$ be the affine Lie algebra of type $A_{1}^{(1)}$ (without a degree operator). Here $K$ is the canonical central element. Note that $\mathfrak{s l}_{2}$ and the current algebra $\mathfrak{s l}_{2}[t]$ are naturally regarded as Lie subalgebras of $\widehat{\mathfrak{s l}_{2}}$. Let $\widehat{\mathfrak{h}}=\mathbb{C} h \oplus \mathbb{C} K$, and $\Lambda_{0}, \Lambda_{1} \in \widehat{\mathfrak{h}}^{*}$ be the fundamental weights defined by

$$
\left\langle h, \Lambda_{0}\right\rangle=0, \quad\left\langle h, \Lambda_{1}\right\rangle=1, \quad\left\langle K, \Lambda_{0}\right\rangle=\left\langle K, \Lambda_{1}\right\rangle=1 .
$$

Define a Borel subalgebra $\widehat{\mathfrak{b}} \subseteq \widehat{\mathfrak{s l}_{2}}$ by

$$
\widehat{\mathfrak{b}}=\widehat{\mathfrak{h}} \oplus \mathbb{C} e \oplus t \mathfrak{s l}_{2}[t] .
$$

Let $\widehat{\mathfrak{p}}_{1}$ and $\widehat{\mathfrak{p}}_{0}$ be the Lie subalgebras of $\widehat{\mathfrak{s l}}$ defined respectively by

$$
\widehat{\mathfrak{p}}_{1}=\mathbb{C} f \oplus \widehat{\mathfrak{b}}=\mathfrak{s l}_{2}[t] \oplus \mathbb{C} K, \quad \widehat{\mathfrak{p}}_{0}=\mathbb{C}\left(e \otimes t^{-1}\right) \oplus \widehat{\mathfrak{b}},
$$

which are minimal parabolic subalgebras. Let $\tau$ be the Lie algebra automorphism on $\widehat{\mathfrak{s l}_{2}}$ induced from the unique nontrivial Dynkin diagram automorphism. Explicitly, $\tau$ is defined as follows:

$$
\tau\left(e \otimes t^{k}\right)=f \otimes t^{k+1}, \quad \tau\left(h \otimes t^{k}\right)=-h \otimes t^{k}+\delta_{k 0} K, \quad \tau(K)=K, \quad \tau^{2}=\text { id. }
$$

Given a finite-dimensional $\widehat{\mathfrak{p}}_{1}$-module $D$ which is $\widehat{\mathfrak{h}}$-semisimple, we define a new $\widehat{\mathfrak{p}}_{1-}$ module $F(D)$ as follows. Let $\tau^{*} D$ be the pull-back with respect to $\tau$, which is a $\widehat{\mathfrak{p}}_{0^{-}}$ module since $\tau\left(\widehat{\mathfrak{p}}_{0}\right)=\widehat{\mathfrak{p}}_{1}$. We consider $\tau^{*} D$ as a $\widehat{\mathfrak{b}}$-module by restriction, and then $F(D)$ is defined by the unique maximal finite-dimensional $\widehat{\mathfrak{p}}_{1}$-module quotient of the induced module $U\left(\widehat{\mathfrak{p}}_{1}\right) \otimes_{U(\widehat{\mathfrak{b}})} \tau^{*}(D)$ (which exists by [FL99, Proposition 2.1]).

For $\ell \in \mathbb{C}$, denote by $\mathbb{C}_{\ell \Lambda_{0}}$ the 1 -dimensional $\widehat{\mathfrak{p}}_{1}$-module on which $K$ acts as a scalar multiplication by $\ell$ and $\mathfrak{s l}_{2}[t]$ acts trivially. Now the following lemma is a reformulation of [FL99, Theorem 2.5] (for the present formulation, see [Nao12a, Theorem 6.1]).

Proposition 5.3. Given a partition $\left(\ell_{1} \geq \cdots \geq \ell_{p-1} \geq \ell_{p}\right)$, it follows that

$$
W^{\ell_{1}} * \cdots * W^{\ell_{p-1}} * W^{\ell_{p}} \cong F\left(\mathbb{C}_{\left(\ell_{1}-\ell_{2}\right) \Lambda_{0}} \otimes \cdots \otimes F\left(\mathbb{C}_{\left(\ell_{p-1}-\ell_{p}\right) \Lambda_{0}} \otimes F\left(\mathbb{C}_{\ell_{p} \Lambda_{0}}\right)\right) \cdots\right)
$$

as $\mathfrak{s l}_{2}[t]$-modules.

We need a slightly alternative realization. Let $\widehat{V}_{0}$ (resp. $\widehat{V}_{1}$ ) be the simple highest weight $\widehat{\mathfrak{s l}_{2}}$-module with highest weight $\Lambda_{0}$ (resp. $\Lambda_{1}$ ). Let $m \in \mathbb{Z}_{\geq 0}$. If $m$ is even (resp. odd), let $v_{m}$ denote an extremal weight vector of $\widehat{V}_{0}$ (resp. $\left.\widehat{V}_{1}\right)$ with weight $m \Lambda_{1}-(m-$ 1) $\Lambda_{0}$. Note that $\tau^{*}\left(V_{0}\right) \cong V_{1}$ and $\tau^{*}\left(V_{1}\right) \cong V_{0}$ hold, and these isomorphisms map $\tau^{*}\left(v_{m}\right)$ to an extremal weight vector with weight $m \Lambda_{0}-(m-1) \Lambda_{1}$, which we denote by $v_{m}^{-}$. It is easily checked that

$$
(f \otimes t) v_{m}^{-}=0, \quad\left(e \otimes t^{-1}\right)^{m} v_{m}^{-} \in \mathbb{C}^{\times} v_{m+1}, \quad\left(e \otimes t^{-1}\right)^{m+1} v_{m}^{-}=0 .
$$

For a sequence $m_{1}, \ldots, m_{p}$ of positive integers, define an $\mathfrak{s l}_{2}[t]$-module $D\left(m_{1}, \ldots, m_{p}\right)$ by

$$
D\left(m_{1}, \ldots, m_{p}\right)=U\left(\mathfrak{s l}_{2}[t]\right)\left(v_{m_{1}} \otimes \cdots \otimes v_{m_{p}}\right) \subseteq \widehat{V}_{\bar{m}_{1}} \otimes \cdots \otimes \widehat{V}_{\bar{m}_{p}},
$$

where $\bar{m}$ is 0 if $m$ is even, and 1 if $m$ is odd. 
Lemma 5.4. For a sequence $\ell=\left(\ell_{1}, \ldots, \ell_{p}\right)$ of positive integers, set

$$
L=\max \left\{\ell_{1}, \ldots, \ell_{p}\right\} \text { and } m_{j}=\#\left\{1 \leq k \leq p \mid \ell_{k} \geq j\right\} \text { for } 1 \leq j \leq L .
$$

Then we have

$$
W^{\ell_{1}} * \cdots * W^{\ell_{p}} \cong D\left(m_{1}, \ldots, m_{L}\right)
$$

as $\mathfrak{s l}_{2}[t]$-modules.

Proof. Without loss of generality we may assume that $\ell$ is a partition, and then by Proposition 5.3 it suffices to show that

$$
F\left(\mathbb{C}_{\left(\ell_{1}-\ell_{2}\right) \Lambda_{0}} \otimes \cdots \otimes F\left(\mathbb{C}_{\left(\ell_{p-1}-\ell_{p}\right) \Lambda_{0}} \otimes F\left(\mathbb{C}_{\ell_{p} \Lambda_{0}}\right)\right) \cdots\right) \cong D\left({ }^{t} \ell\right),
$$

where ${ }^{t} \boldsymbol{\ell}$ is the transposed partition. We will show this by the induction on $p$. The case $p=1$ is easily checked. Assume $p>1$, and set $\ell^{\prime}=\left(\ell_{2}, \ldots, \ell_{p}\right)$. Since

$$
D\left({ }^{t} \ell^{\prime}\right)=U\left(\widehat{\mathfrak{p}}_{1}\right)\left(v_{m_{1}-1} \otimes \cdots \otimes v_{m_{\ell_{2}}-1}\right),
$$

we have

$$
\begin{aligned}
\tau^{*}\left(\mathbb{C}_{\left(\ell_{1}-\ell_{2}\right) \Lambda_{0}} \otimes D\left({ }^{t} \ell^{\prime}\right)\right) & \cong \tau^{*}\left(U\left(\widehat{\mathfrak{p}}_{1}\right)\left(v_{0}^{\otimes\left(\ell_{1}-\ell_{2}\right)} \otimes v_{m_{1}-1} \otimes \cdots \otimes v_{m_{\ell_{2}}-1}\right)\right) \\
& \cong U\left(\widehat{\mathfrak{p}}_{0}\right)\left(v_{1}^{\otimes\left(\ell_{1}-\ell_{2}\right)} \otimes v_{m_{1}-1}^{-} \otimes \cdots \otimes v_{m_{\ell_{2}}-1}^{-}\right) \\
& =U(\widehat{\mathfrak{b}})\left(v_{1}^{\otimes\left(\ell_{1}-\ell_{2}\right)} \otimes v_{m_{1}} \otimes \cdots \otimes v_{m_{\ell_{2}}}\right)
\end{aligned}
$$

where the equality follows from (5.1). Hence by the definition of $F$, there exists a surjective $\widehat{\mathfrak{p}}_{1}$-module homomorphism

$$
F\left(\mathbb{C}_{\left(\ell_{1}-\ell_{2}\right) \Lambda_{0}} \otimes D\left({ }^{t} \boldsymbol{\ell}^{\prime}\right)\right) \rightarrow U\left(\widehat{\mathfrak{p}}_{1}\right)\left(v_{1}^{\otimes\left(\ell_{1}-\ell_{2}\right)} \otimes v_{m_{1}} \otimes \cdots \otimes v_{m_{\ell_{2}}}\right) \cong D\left({ }^{t} \boldsymbol{\ell}\right),
$$

which induces a surjection

$$
F\left(\mathbb{C}_{\left(\ell_{1}-\ell_{2}\right) \Lambda_{0}} \otimes \cdots \otimes F\left(\mathbb{C}_{\left(\ell_{p-1}-\ell_{p}\right) \Lambda_{0}} \otimes F\left(\mathbb{C}_{\ell_{p} \Lambda_{0}}\right)\right) \cdots\right) \rightarrow D\left({ }^{t} \boldsymbol{\ell}\right)
$$

by the induction hypothesis. Since the dimensions of these modules coincide by LLM02, Theorem 5] and [Nao12a, Corollary 6.2], this is an isomorphism. Hence (5.2) is proved, as required.

Finally we recall the following proposition.

Proposition 5.5. Assume that $a_{1}, \ldots, a_{p} \in \mathcal{A}^{\times}$satisfy $a_{1}(1)=\cdots=a_{p}(1)=c \in \mathbb{C}^{\times}$, and $W_{q}^{1}\left(a_{1}\right) \otimes \cdots \otimes W_{q}^{1}\left(a_{p}\right)$ is $\ell$-highest weight. Then we have

$$
\overline{W_{q}^{1}\left(a_{1}\right) \otimes \cdots \otimes W_{q}^{1}\left(a_{p}\right)} \cong \varphi_{c}^{*} D(p)
$$

as $\mathfrak{s l}_{2}[t]$-modules.

Proof. This follows from CP01, Theorem 5] and [CL06, Corollary 1.5.1]. 
5.3. Proof of Proposition 3.5. As in Proposition 3.5, let $\ell_{1}, \ldots, \ell_{p} \in \mathbb{Z}_{>0}$ and $a_{1}, \ldots, a_{p} \in \mathcal{A}^{\times}$be such that $a_{1}(1)=\cdots=a_{p}(1)=c \in \mathbb{C}^{\times}$and $W_{q}^{\ell_{1}}\left(a_{1}\right) \otimes \cdots \otimes W_{q}^{\ell_{p}}\left(a_{p}\right)$ is $\ell$-highest weight.

Lemma 5.6. There exists a permutation $\sigma$ on the set $\{1, \ldots, p\}$ satisfying

$$
a_{\sigma(s)} / a_{\sigma(r)} \notin \tilde{q}^{2 \mathbb{Z}_{>0}} \quad \text { for all } 1 \leq r<s \leq p,
$$

and

$$
W_{q}^{\ell_{1}}\left(a_{1}\right) \otimes \cdots \otimes W_{q}^{\ell_{p}}\left(a_{p}\right) \cong W_{q}^{\ell_{\sigma(1)}}\left(a_{\sigma(1)}\right) \otimes \cdots \otimes W_{q}^{\ell_{\sigma(p)}}\left(a_{\sigma(p)}\right) .
$$

Proof. We show the assertion by the induction on $p$. There is nothing to prove when $p=1$. Assume that $p>1$. By the induction hypothesis, we may assume that

$$
a_{s} / a_{r} \notin \tilde{q}^{2 \mathbb{Z}_{>0}} \text { for } 1 \leq r<s \leq p-1 .
$$

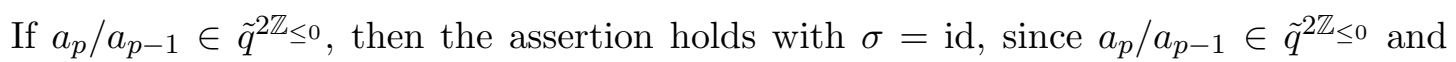
$a_{p-1} / a_{r} \notin \tilde{q}^{2 \mathbb{Z}_{>0}}$ imply $a_{p} / a_{r} \notin \tilde{q}^{2 \mathbb{Z}_{>0}}$. Assume that $a_{p} / a_{p-1} \notin \tilde{q}^{2 \mathbb{Z}_{\leq 0}}$. If

$$
a_{p} / a_{p-1} \in\left\{\tilde{q}^{2 k} \mid \max \left\{\ell_{p-1}-\ell_{p}, 0\right\}<k \leq \ell_{p}\right\},
$$

then the submodule of $W_{q}^{\ell_{p-1}}\left(a_{p-1}\right) \otimes W_{q}^{\ell_{p}}\left(a_{p}\right)$ generated by the tensor product of $\ell$ highest weight vectors is proper by Lemma 5.1 (ii), which contradicts that $W_{q}^{\ell_{1}}\left(a_{1}\right) \otimes$ $\cdots \otimes W_{q}^{\ell_{p}}\left(a_{p}\right)$ is $\ell$-highest weight. Hence $W_{q}^{\ell_{p-1}}\left(a_{p-1}\right) \otimes W_{q}^{\ell_{p}}\left(a_{p}\right)$ is simple by Lemma 5.1 (iii), and we have

$$
W_{q}^{\ell_{1}}\left(a_{1}\right) \otimes \cdots \otimes W_{q}^{\ell_{p-1}}\left(a_{p-1}\right) \otimes W_{q}^{\ell_{p}}\left(a_{p}\right) \cong W_{q}^{\ell_{1}}\left(a_{1}\right) \otimes \cdots \otimes W_{q}^{\ell_{p}}\left(a_{p}\right) \otimes W_{q}^{\ell_{p-1}}\left(a_{p-1}\right) .
$$

Now by applying the induction hypothesis to $W_{q}^{\ell_{1}}\left(a_{1}\right) \otimes \cdots \otimes W_{q}^{\ell_{p-2}}\left(a_{p-2}\right) \otimes W_{q}^{\ell_{p}}\left(a_{p}\right)$, we obtain the required result.

By this lemma, we may (and do) assume that

$$
a_{s} / a_{r} \notin \tilde{q}^{2 \mathbb{Z}_{>0}} \text { for all } 1 \leq r<s \leq p .
$$

Put $L=\max \left\{\ell_{k} \mid 1 \leq k \leq p\right\}$, and

$$
M_{j}=\left\{1 \leq k \leq p \mid \ell_{k} \geq j\right\} \text { for } 1 \leq j \leq L .
$$

Lemma 5.7. There exists an injective $U_{q}\left(\mathbf{L s t}_{2}\right)$-module homomorphism

$$
\iota: W_{q}^{\ell_{1}}\left(a_{1}\right) \otimes \cdots \otimes W_{q}^{\ell_{p}}\left(a_{p}\right) \hookrightarrow \bigotimes_{k \in M_{1}} W_{q}^{1}\left(a_{k}\right) \otimes \bigotimes_{k \in M_{2}} W_{q}^{1}\left(\tilde{q}^{2} a_{k}\right) \otimes \cdots \otimes \bigotimes_{k \in M_{L}} W_{q}^{1}\left(\tilde{q}^{2 L-2} a_{k}\right)
$$

mapping an $\ell$-highest weight vector to a tensor product of $\ell$-highest weight vectors. Here each $\bigotimes_{k} W_{q}^{1}\left(\tilde{q}^{2 j} a_{k}\right)$ are ordered so that $W_{q}^{1}\left(\tilde{q}^{2 j} a_{r}\right)$ is left to $W_{q}^{1}\left(\tilde{q}^{2 j} a_{s}\right)$ if $r<s$.

Proof. We show the assertion by the induction on $p$. If $p=1$, it follows from Lemma 5.1 (iv). Assume that $p>1$. We claim that, for each $1 \leq j \leq \ell_{1}-1$, there exists an injective homomorphism

$$
W_{q}^{\ell_{1}+1-j}\left(\tilde{q}^{2 j-2} a_{1}\right) \otimes \bigotimes_{k \in M_{j} \backslash\{1\}} W_{q}^{1}\left(\tilde{q}^{2 j-2} a_{k}\right) \hookrightarrow \bigotimes_{k \in M_{j}} W_{q}^{1}\left(\tilde{q}^{2 j-2} a_{k}\right) \otimes W_{q}^{\ell_{1}-j}\left(\tilde{q}^{2 j} a_{1}\right) .
$$


Indeed, putting $T=\left\{k \in M_{j} \mid a_{k}=a_{1}\right\}$, it follows from (5.3) and Lemma 5.1 that

$$
\begin{aligned}
W_{q}^{\ell_{1}+1-j} & \left(\tilde{q}^{2 j-2} a_{1}\right) \otimes \bigotimes_{k \in M_{j} \backslash\{1\}} W_{q}^{1}\left(\tilde{q}^{2 j-2} a_{k}\right) \\
& \stackrel{\sim}{\rightarrow} W_{q}^{1}\left(\tilde{q}^{2 j-2} a_{1}\right)^{\otimes(\# T-1)} \otimes W_{q}^{\ell_{1}+1-j}\left(\tilde{q}^{2 j-2} a_{1}\right) \otimes \bigotimes_{k \in M_{j} \backslash T} W_{q}^{1}\left(\tilde{q}^{2 j-2} a_{k}\right) \\
& \hookrightarrow W_{q}^{1}\left(\tilde{q}^{2 j-2} a_{1}\right)^{\otimes \# T} \otimes W_{q}^{\ell_{1}-j}\left(\tilde{q}^{2 j} a_{1}\right) \otimes \bigotimes_{k \in M_{j} \backslash T} W_{q}^{1}\left(\tilde{q}^{2 j-2} a_{k}\right) \\
& \stackrel{\sim}{\rightarrow} \bigotimes_{k \in M_{j}} W_{q}^{1}\left(\tilde{q}^{2 j-2} a_{k}\right) \otimes W_{q}^{\ell_{1}-j}\left(\tilde{q}^{2 j} a_{1}\right),
\end{aligned}
$$

and hence the claim is proved.

By composing the homomorphisms induced from (5.4), we obtain an injective homomorphism

$$
\begin{aligned}
W_{q}^{\ell_{1}}\left(a_{1}\right) \otimes \bigotimes_{k \in M_{1} \backslash\{1\}} W_{q}^{1}\left(a_{k}\right) \otimes & \cdots \otimes \bigotimes_{k \in M_{L} \backslash\{1\}} W_{q}^{1}\left(\tilde{q}^{2 L-2} a_{k}\right) \\
& \hookrightarrow \bigotimes_{k \in M_{1}} W_{q}^{1}\left(a_{k}\right) \otimes \cdots \otimes \bigotimes_{k \in M_{L}} W_{q}^{1}\left(\tilde{q}^{2 L-2} a_{k}\right) .
\end{aligned}
$$

Since there is an injective homomorphism from $W_{q}^{\ell_{1}}\left(a_{1}\right) \otimes \cdots \otimes W_{q}^{\ell_{p}}\left(a_{p}\right)$ to the left-hand side by the induction hypothesis, the assertion is proved.

Note that, by Lemma 5.2 and (5.3), $\bigotimes_{k \in M_{j}} W_{q}^{1}\left(\tilde{q}^{2 j-2} a_{k}\right)$ are $\ell$-highest weight for all $j$. Let $v \in W_{q}^{\ell_{1}}\left(a_{1}\right) \otimes \cdots \otimes W_{q}^{\ell_{p}}\left(a_{p}\right)$ and $v_{j} \in \bigotimes_{k \in M_{j}} W_{q}\left(\tilde{q}^{2 j-2} a_{k}\right)$ be $\ell$-highest weight vectors satisfying $\iota(v)=v_{1} \otimes \cdots \otimes v_{\ell_{L}}$. By Lemma 2.5 we have

$$
\iota\left(U_{\mathcal{A}}\left(\mathbf{L s l}_{2}\right) v\right) \subseteq U_{\mathcal{A}}\left(\mathbf{L s t}_{2}\right) v_{1} \otimes \cdots \otimes U_{\mathcal{A}}\left(\mathbf{L s l}_{2}\right) v_{\ell_{L}},
$$

and hence $\iota$ induces an $\mathbf{L s l}_{2}$-module homomorphism

$$
\bar{\iota}: \overline{W_{q}^{\ell_{1}}\left(a_{1}\right) \otimes \cdots \otimes W_{q}^{\ell_{p}}\left(a_{p}\right)} \rightarrow \overline{\bigotimes_{k \in M_{1}} W_{q}^{1}\left(a_{k}\right)} \otimes \cdots \otimes \overline{\bigotimes_{k \in M_{\ell_{L}}} W_{q}^{1}\left(\tilde{q}^{2 \ell_{L}-2} a_{k}\right)} .
$$

Set $m_{j}=\# M_{j}(1 \leq j \leq L)$. By Proposition 5.5, the right-hand side is isomorphic to

$$
\varphi_{c}^{*} D\left(m_{1}\right) \otimes \cdots \otimes \varphi_{c}^{*} D\left(m_{L}\right)=\varphi_{c}^{*}\left(D\left(m_{1}\right) \otimes \cdots \otimes D\left(m_{L}\right)\right),
$$

and the image of the composition of this isomorphism with $\bar{\iota}$ is $\varphi_{c}^{*} D\left(m_{1}, \ldots, m_{L}\right)$, which is isomorphic to $\varphi_{c}^{*}\left(W^{\ell_{1}} * \cdots * W^{\ell_{p}}\right)$ by Lemma 5.4. Hence we obtain a surjective homomorphism

$$
\overline{W_{q}^{\ell_{1}}\left(a_{1}\right) \otimes \cdots \otimes W_{q}^{\ell_{p}}\left(a_{p}\right)} \rightarrow \varphi_{c}^{*}\left(W^{\ell_{1}} * \cdots * W^{\ell_{p}}\right),
$$

and it is easy to see that the dimensions of the two modules are equal. Hence Proposition 3.5 is proved. 


\section{Appendix A.}

In this appendix, we will give proofs of the results mentioned in Section 4 . We use freely the notations introduced in the section. For $\gamma=\sum_{i} m_{i} \alpha_{i} \in Q^{+}$we write

$$
\Delta_{\gamma}=\prod_{i<j} \prod_{\substack{1 \leq r \leq m_{i} \\ 1 \leq s \leq m_{j}}}\left(x_{r}^{(i)}-x_{s}^{(j)}\right)
$$

for ease of notation.

A.1. Filtration on $\mathcal{U}_{\gamma}$. Let $\gamma=\sum_{i} m_{i} \alpha_{i} \in Q^{+}$. Following [AK07, we will define a filtration on $\mathcal{U}_{\gamma}$, which plays an important roll in the next subsections.

Let $\boldsymbol{\mu}=\left(\mu^{(i)}\right)_{i \in I}$ be an $I$-tuple of partitions satisfying $\left|\mu^{(i)}\right|=m_{i}$, and denote by $m_{a}^{(i)}$ the number of rows of length $a$ in $\mu^{(i)}$ (here, as usual, we identify partitions with Young diagrams). Let $\boldsymbol{y}_{\boldsymbol{\mu}}$ be the set of variables indexed by the rows of $\mu^{(i)}$ 's:

$$
\boldsymbol{y}_{\boldsymbol{\mu}}=\left\{y_{a, u}^{(i)} \mid i \in I, a>0,1 \leq u \leq m_{a}^{(i)}\right\} .
$$

We will define a specialization map $\varphi_{\boldsymbol{\mu}}$ from $\mathcal{U}_{\gamma}$ to the space of rational functions $\mathbb{C}\left(\boldsymbol{y}_{\boldsymbol{\mu}}\right)$ in $\boldsymbol{y}_{\boldsymbol{\mu}}$. For each $i \in I$, reindex (arbitrarily) the variables $\left\{x_{r}^{(i)} \mid 1 \leq r \leq m_{i}\right\}$ into

$$
\left\{x_{a, u, v}^{(i)} \mid a>0,1 \leq u \leq m_{a}^{(i)}, 1 \leq v \leq a\right\}
$$

which are parametrized by the boxes of $\mu^{(i)}$. Then let $\varphi_{\boldsymbol{\mu}}: \mathcal{U}_{\gamma} \rightarrow \mathbb{C}\left(\boldsymbol{y}_{\boldsymbol{\mu}}\right)$ be the linear homomorphism naturally defined from the map

$$
\boldsymbol{x}_{\gamma} \rightarrow \boldsymbol{y}_{\boldsymbol{\mu}}: x_{a, u, v}^{(i)} \mapsto y_{a, u}^{(i)},
$$

which does not depend on the reindexing due to the symmetry.

Define a lexicographic ordering $\leq$ on the set of $I$-tuples of partitions by $\boldsymbol{\mu}<\boldsymbol{\nu}$ if and only if there exists $i \in I$ such that $\mu^{(j)}=\nu^{(j)}$ for $j<i$ and $\mu^{(i)}<\nu^{(i)}$. Here the ordering on partitions are the usual lexicographic one. Let

$$
\Gamma_{\boldsymbol{\mu}}=\bigcap_{\boldsymbol{\nu}>\boldsymbol{\mu}} \operatorname{ker} \varphi_{\boldsymbol{\nu}} \subseteq \mathcal{U}_{\gamma}
$$

which defines a filtration $\mathcal{U}_{\gamma}=\bigcup_{\boldsymbol{\mu}} \Gamma_{\boldsymbol{\mu}}$. We also define $\Gamma_{\boldsymbol{\mu}}^{\prime}=\bigcap_{\boldsymbol{\nu} \geq \boldsymbol{\mu}} \operatorname{ker} \varphi_{\boldsymbol{\nu}} \subseteq \mathcal{U}_{\gamma}$. The zeros and poles of the functions in the image $\varphi_{\boldsymbol{\mu}}\left(\Gamma_{\boldsymbol{\mu}}\right) \cong \Gamma_{\boldsymbol{\mu}} / \Gamma_{\boldsymbol{\mu}}^{\prime}$ are described by the following lemma. For the proof, see [AK07, Appendix A].

Lemma A.1. Assume that $g(\boldsymbol{x}) \in \Gamma_{\boldsymbol{\mu}}$.

(i) The function $\varphi_{\boldsymbol{\mu}}(g(\boldsymbol{x}))$ has a zero of order at least $2 \min \{a, b\}$ whenever $y_{a, u}^{(i)}=y_{b, v}^{(i)}$.

(ii) For $i, j \in I$ with $i \neq j, \varphi_{\boldsymbol{\mu}}(g(\boldsymbol{x}))$ has a pole of order at most $\min \left\{\left|c_{j i}\right| a,\left|c_{i j}\right| b\right\}$ whenever $y_{a, u}^{(i)}=y_{b, v}^{(j)}$.

By this lemma, we see that $\varphi_{\boldsymbol{\mu}}(g(\boldsymbol{x}))$ for $g(\boldsymbol{x}) \in \Gamma_{\boldsymbol{\mu}}$ is of the form

$$
\frac{\prod_{i \in I} \prod_{(a, u)<(b, v)}\left(y_{a, u}^{(i)}-y_{b, v}^{(i)}\right)^{2 \min \{a, b\}}}{\prod_{i<j} \prod_{(a, u),(b, v)}\left(y_{a, u}^{(i)}-y_{b, v}^{(j)}\right)^{\min \left\{\left|c_{j i}\right| a,\left|c_{i j}\right| b\right\}}} \cdot h_{0}\left(\boldsymbol{y}_{\boldsymbol{\mu}}\right),
$$

where $h_{0}\left(\boldsymbol{y}_{\boldsymbol{\mu}}\right)$ is a Laurent polynomial in $\boldsymbol{y}_{\boldsymbol{\mu}}$ and symmetric under the exchange of variables $y_{a, u}^{(i)} \leftrightarrow y_{a, v}^{(i)}$. 
A.2. Simplicity of poles. The purpose of this subsection is to prove the following lemma.

Lemma A.2. Let $\gamma \in Q^{+}$, and $i_{1}, i_{2}, \ldots$ be a sequence of elements of $I$. Define a sequence of variables $x_{1}, x_{2}, \ldots$ by $x_{r}=x_{\#\left\{s \leq r \mid i_{s}=i_{r}\right\}}^{\left(i_{r}\right)}$. Take $g_{1}(\boldsymbol{x}) \in \mathcal{U}_{\gamma}$ arbitrarily, and define functions $g_{r}(\boldsymbol{x})(r=1,2, \ldots)$ inductively by

$$
g_{r}(\boldsymbol{x})=\operatorname{Res}_{x_{r-1}=x_{r}} g_{r-1}(\boldsymbol{x}) .
$$

Then each $g_{r}(\boldsymbol{x})$ has at most a simple pole at $x_{r}=x_{r+1}$.

Let $\mathbb{C}\left[\boldsymbol{x}_{\gamma}\right]$ denote the polynomial algebra in $\boldsymbol{x}_{\gamma}$, and define a subspace $\mathcal{U}_{\gamma}^{\prime} \subseteq \mathcal{U}_{\gamma}$ by

$$
\mathcal{U}_{\gamma}^{\prime}=\mathcal{U}_{\gamma} \cap\left\{g^{\prime}(\boldsymbol{x}) / \Delta_{\gamma} \mid g^{\prime}(\boldsymbol{x}) \in \mathbb{C}\left[\boldsymbol{x}_{\gamma}\right]\right\} .
$$

Obviously $\mathcal{U}_{\gamma}^{\prime}$ is a $\mathbb{Z}$-graded subspace with respect to the total degree.

Lemma A.3. Assume that $\gamma \neq 0$ and $g(\boldsymbol{x}) \in \mathcal{U}_{\gamma}^{\prime}$ is homogeneous. Then we have

$$
\operatorname{deg} g(\boldsymbol{x})>-\operatorname{ht}(\gamma) \text {. }
$$

Proof. Take $\boldsymbol{\mu}=\left(\mu^{(1)}, \ldots, \mu^{(n)}\right)$ so that $g(\boldsymbol{x}) \in \Gamma_{\boldsymbol{\mu}} \backslash \Gamma_{\boldsymbol{\mu}}^{\prime}$, and let $m_{a}^{(i)}$ be the number of rows of length $a$ in $\mu^{(i)}$. The image $\varphi_{\mu}\left(\Gamma_{\boldsymbol{\mu}}\right)$ is also $\mathbb{Z}$-graded by the total degree, and it is enough to show that

$$
\operatorname{deg} \varphi_{\boldsymbol{\mu}}(g(\boldsymbol{x}))>-\operatorname{ht}(\gamma)
$$

since $\varphi_{\boldsymbol{\mu}}$ preserves the degree. Since $g(\boldsymbol{x}) \in \mathcal{U}_{\gamma}^{\prime}$, it is clear that $\varphi_{\boldsymbol{\mu}}(g(\boldsymbol{x}))$ is of the form (A.1) with $h_{0}\left(\boldsymbol{y}_{\boldsymbol{\mu}}\right)$ being a polynomial. Hence we have

$$
\operatorname{deg} \varphi_{\boldsymbol{\mu}}(g(\boldsymbol{x})) \geq 2 \sum_{i \in I} \sum_{(a, u)<(b, v)} \min \{a, b\}-\sum_{i<j} \sum_{(a, u),(b, v)} \min \left\{\left|c_{j i}\right| a,\left|c_{i j}\right| b\right\},
$$

and the right-hand side is larger than $-\operatorname{ht}(\gamma)$ by Lemma A.4 given below. Hence the assertion is proved.

Proof of Lemma A.9. We show the lemma by the induction on $r$. The case $r=1$ is trivial. Assume that $r>1$, and set $\beta=\alpha_{i_{1}}+\alpha_{i_{2}}+\cdots+\alpha_{i_{r+1}}$. We may assume that $\gamma-\beta \in Q^{+}$, since otherwise $g_{r}(\boldsymbol{x})$ does not contain the variable $x_{r+1}$ and hence the assertion trivially holds. Then, since

$$
\mathcal{U}_{\gamma} \subseteq \mathbb{C}\left[x,\left(x-x^{\prime}\right)^{-1} \mid x \in \boldsymbol{x}_{\gamma} \backslash \boldsymbol{x}_{\beta}, x^{\prime} \in \boldsymbol{x}_{\gamma}\right] \cdot \mathcal{U}_{\beta},
$$

it is enough to show the assertion in the case $\gamma=\beta$. Therefore we assume $\gamma=\beta$ in the sequel.

Without loss of generality we may assume that $g_{1}(\boldsymbol{x})$ is homogeneous, and since multiplying a monomial $\left(x_{1} x_{2} \ldots x_{r+1}\right)^{a}$ preserves $\mathcal{U}_{\beta}$ and does not affect the orders of the poles we are considering, we may further assume that $g_{1}(\boldsymbol{x}) \in \mathcal{U}_{\beta}^{\prime}$. Write $g_{1}(\boldsymbol{x})=$ $g_{1}^{\prime}(\boldsymbol{x}) / \Delta_{\beta}$, and set $N=\operatorname{deg} g_{1}^{\prime}(\boldsymbol{x})$.

Let $\mathbb{C}\left[\boldsymbol{x}_{\beta}\right]^{\prime}$ denote the $\mathbb{C}$-subalgebra of $\mathbb{C}\left[\boldsymbol{x}_{\beta}\right]$ generated by the vectors $\left(x_{k}-x_{l}\right)$ for $1 \leq k<l \leq r+1$. Since $\mathbb{C}\left[\boldsymbol{x}_{\beta}\right]=\mathbb{C}\left[x_{r+1}\right] \cdot \mathbb{C}\left[\boldsymbol{x}_{\beta}\right]^{\prime}$ holds, $g_{1}^{\prime}(\boldsymbol{x})$ is uniquely written as

$$
g_{1}^{\prime}(\boldsymbol{x})=\sum_{k=k_{0}}^{N} x_{r+1}^{N-k} q_{k}(\boldsymbol{x}),
$$


where $q_{k}(\boldsymbol{x})$ is a homogeneous polynomial in $\mathbb{C}\left[\boldsymbol{x}_{\beta}\right]^{\prime}$ with degree $k$, and $q_{k_{0}}(\boldsymbol{x}) \neq 0$. We claim that $q_{k_{0}}(\boldsymbol{x}) / \Delta_{\beta} \in \mathcal{U}_{\beta}^{\prime}$. Indeed, it is easy to check that $\left.g_{1}^{\prime}(\boldsymbol{x})\right|_{x_{1}^{(i)}=\cdots=x_{\bar{c}_{i j}}^{(i)}=x_{1}^{(j)}}=0$ implies $\left.q_{k}(\boldsymbol{x})\right|_{x_{1}^{(i)}=\cdots=x_{\bar{c}_{i j}}^{(i)}=x_{1}^{(j)}}=0$ for all $k$. Moreover, the symmetry of $g_{1}^{\prime}(\boldsymbol{x})$ under the exchange of variables $x_{s}^{(i)} \leftrightarrow x_{s^{\prime}}^{(i)}$ implies the same symmetry on $q_{k_{0}}(\boldsymbol{x})$, since

$$
x_{s}^{N-k}=\left(x_{r+1}-\left(x_{r+1}-x_{s}\right)\right)^{N-k} \in x_{r+1}^{N-k}+\mathbb{C}\left[x_{r+1}\right] \cdot\left(\mathbb{C}\left[\boldsymbol{x}_{\beta}\right]^{\prime}\right)^{>0} .
$$

Hence the claim is proved.

Therefore we have $\operatorname{deg}\left(q_{k_{0}}(\boldsymbol{x}) / \Delta_{\beta}\right) \geq-r$ by Lemma A.3, which implies

$$
\operatorname{deg}\left(q_{k}(\boldsymbol{x}) / \Delta_{\beta}\right) \geq-r \text { for all } k \text { such that } q_{k}(\boldsymbol{x}) \neq 0 \text {. }
$$

Note that, by the induction hypothesis, we have

$$
g_{r}(\boldsymbol{x})=\left.\prod_{s=2}^{r}\left(x_{s-1}-x_{s}\right) g_{1}(\boldsymbol{x})\right|_{x_{1}=\cdots=x_{r}}=\left.\sum_{k=k_{0}}^{N} x_{r+1}^{N-k}\left(\prod_{s=2}^{r}\left(x_{s-1}-x_{s}\right) q_{k}(\boldsymbol{x}) / \Delta_{\beta}\right)\right|_{x_{1}=\cdots=x_{r}} .
$$

Since $q_{k}(\boldsymbol{x}) \in \mathbb{C}\left[\boldsymbol{x}_{\beta}\right]^{\prime}$, we have

$$
\left.\left(\prod_{s=2}^{r}\left(x_{s-1}-x_{s}\right) q_{k}(\boldsymbol{x}) / \Delta_{\beta}\right)\right|_{x_{1}=\cdots=x_{r}} \in \mathbb{C}\left[\left(x_{r}-x_{r+1}\right)^{ \pm 1}\right]
$$

for all $k$, and its degree is equal to or more than -1 by (A.2). Hence the assertion is proved.

It remains to prove the following.

Lemma A.4. Let $\boldsymbol{\mu}=\left(\mu^{(1)}, \ldots, \mu^{(n)}\right)$ be an I-tuple of partitions such that $\mu^{(i)}$ has $m_{a}^{(i)}$ rows of length $a$, and assume that at least one of the partitions is nonzero. Set $R\left(\mu^{(i)}\right)=\left\{(a, u) \mid a>0,1 \leq u \leq m_{a}^{(i)}\right\}$, and define

$$
P(\boldsymbol{\mu})=2 \sum_{i \in I} \sum_{\substack{(a, u),(b, v) \in R\left(\mu^{(i)}\right) ; \\(a, u)<(b, v)}} \min \{a, b\}-\sum_{i<j} \sum_{\substack{(a, u) \in R\left(\mu^{(i)}\right) \\(b, v) \in R\left(\mu^{(j)}\right)}} \min \left\{\left|c_{j i}\right| a,\left|c_{i j}\right| b\right\} .
$$

Then we have $P(\boldsymbol{\mu})>-\sum_{i}\left|\mu^{(i)}\right|$.

Proof. We prove the assertion by the induction on $\sum_{i}\left|\mu^{(i)}\right|$. The case $\sum_{i}\left|\mu^{(i)}\right|=1$ is easily checked.

Assume that $\sum_{i}\left|\mu^{(i)}\right|>1$. For $i \in I$, set

$$
c_{i}= \begin{cases}1 & \text { if } \alpha_{i} \text { is long, } \\ 2 & \text { if } \mathfrak{g} \text { is of type } B C F \text { and } \alpha_{i} \text { is short, } \\ 3 & \text { if } \mathfrak{g} \text { is of type } G_{2} \text { and } \alpha_{i} \text { is short, }\end{cases}
$$

where we say every $\alpha \in R^{+}$is long when $\mathfrak{g}$ is of type $A D E$. Let

$$
L=\max \left\{\left\lceil\mu_{1}^{(i)} / c_{i}\right\rceil \mid i \in I\right\},
$$


where $\lceil a\rceil$ is the smallest integer equal to or larger than $a$. Let $N_{i}(i \in I)$ denote the number of boxes in $\mu^{(i)}$ strictly right to the $c_{i}(L-1)$-th column;

$N_{i}=m_{L}^{(i)}\left(c_{i}=1\right), \quad N_{i}=2 m_{2 L}^{(i)}+m_{2 L-1}^{(i)}\left(c_{i}=2\right), \quad N_{i}=3 m_{3 L}^{(i)}+2 m_{3 L-1}^{(i)}+m_{3 L-2}^{(i)}\left(c_{i}=3\right)$.

Set $\beta=\sum_{i} N_{i} \alpha_{i} \in Q^{+}$. Since $\mathfrak{g}$ is of finite type, there exists $j \in I$ such that $\left\langle\alpha_{j}^{\vee}, \beta\right\rangle>0$. Fix such an index $j$. We claim that there exists a partition $\mu^{\prime}$ of $\left|\mu^{(j)}\right|-1$ such that

$$
P(\boldsymbol{\mu})-P\left(\boldsymbol{\mu}^{\prime}\right) \geq-1,
$$

where $\boldsymbol{\mu}^{\prime}$ is the $I$-tuple of partitions obtained from $\boldsymbol{\mu}$ by replacing $\mu^{(j)}$ with $\mu^{\prime}$. By the induction hypothesis, this completes the proof.

Let us prove the claim. First assume that $c_{j}=1$. Note that $m_{L}^{(j)}>0$ holds since $\left\langle\alpha_{j}^{\vee}, \beta\right\rangle>0$. Let $\mu^{\prime}$ be the partition obtained from $\mu^{(j)}$ by removing one box in the $L$-th column. Then it is directly checked that

$$
P(\boldsymbol{\mu})-P\left(\boldsymbol{\mu}^{\prime}\right)=2\left(m_{L}^{(j)}-1\right)-\sum_{\substack{i \in I ; \\ c_{i j}<0}} N_{i}=\left\langle\alpha_{j}^{\vee}, \beta\right\rangle-2 \geq-1,
$$

where $\boldsymbol{\mu}^{\prime}$ is defined as above. Hence the claim is proved in this case.

Next assume that $c_{j}=2$, and let us further assume that both $m_{2 L-1}^{(j)}$ and $m_{2 L}^{(j)}$ are nonzero. For $k=0,1$, let $\mu^{\prime}[k]$ denote the partition obtained from $\mu^{(j)}$ by removing one box in the $(2 L-k)$-th column, and set $\boldsymbol{\mu}^{\prime}[k]$ to be the $I$-tuple of partitions obtained from $\boldsymbol{\mu}$ by replacing $\mu^{(j)}$ with $\mu^{\prime}[k]$. Then it follows that

$$
P(\boldsymbol{\mu})-P\left(\boldsymbol{\mu}^{\prime}[k]\right)=2\left(m_{2 L}^{(j)}+\delta_{k 1} m_{2 L-1}^{(j)}-1\right)-\sum_{\substack{i \in I ; \\ c_{j i}=-2}} m_{L}^{(i)}-\sum_{\substack{i \in I ; \\ c_{j i}=-1}}\left(m_{2 L}^{(i)}+\delta_{k 1} m_{2 L-1}^{(i)}\right) .
$$

Hence we have

$$
\left(P(\boldsymbol{\mu})-P\left(\boldsymbol{\mu}^{\prime}[0]\right)\right)+\left(P(\boldsymbol{\mu})-P\left(\boldsymbol{\mu}^{\prime}[1]\right)\right)=\left\langle\alpha_{j}^{\vee}, \beta\right\rangle-4>-4,
$$

which implies that there is $k \in\{0,1\}$ such that $P(\boldsymbol{\mu})-P\left(\boldsymbol{\mu}^{\prime}[k]\right) \geq-1$. When $m_{2 L-k}^{(j)}=0$ for either $k=0$ or $k=1$, we can show similarly that $P(\boldsymbol{\mu})-P\left(\boldsymbol{\mu}^{\prime}\left[k^{\prime}\right]\right) \geq-1$ for $k^{\prime} \neq k$. Hence the claim is verified in this case.

The case $c_{j}=3$ is proved similarly, and we omit the detail.

A.3. Proof of Proposition 4.7. We will define functions $g_{\gamma, k}(\boldsymbol{x}) \in \overline{\mathcal{U}}_{\gamma}$ for $\gamma \in R^{+}$and $k \in \mathbb{Z}_{>0}$. Let $($,$) denote the unique nondegenerate W$-invariant symmetric bilinear form on $P$ normalized so that the square length of $\theta$ is 2 .

First assume that $\mathfrak{g}$ is not of type $G_{2}$, and set $I_{\mathrm{sh}}=\left\{i \in I \mid \alpha_{i}\right.$ is short $\}$. Fix $\gamma=\sum_{i} m_{i} \alpha_{i} \in R^{+}$, and write $\bar{\gamma}=\sum_{i \in I_{\text {sh }} ; m_{i} \notin 2 \mathbb{Z}} \alpha_{i}$. It follows that $(\gamma, \gamma)+(\bar{\gamma}, \bar{\gamma})=2$. Let

$$
h_{\gamma}(\boldsymbol{x})=\prod_{i \in I \backslash I_{\mathrm{sh}}} \prod_{r<s}\left(x_{r}^{(i)}-x_{s}^{(i)}\right)^{2} \prod_{i \in I_{\mathrm{sh}}} \prod_{\substack{r<s \\ r, s: \text { even, or } \\ r, s: \text { odd }}}\left(x_{r}^{(i)}-x_{s}^{(i)}\right)^{2} \prod_{\substack{i, j \in I_{\mathrm{sh}} ; \\ i<j, c_{i j}=-1}} \prod_{\substack{r: \text { even, }, s: \text { odd, or } \\ r: \text { odd, } s: \text { even }}}\left(x_{r}^{(i)}-x_{s}^{(j)}\right) .
$$


For $i \in I$ and $1 \leq r \leq m_{i}$, put

$$
\gamma^{(i, r)}= \begin{cases}\gamma-(-1)^{r} \bar{\gamma} & \text { if } i \in I_{\mathrm{sh}} \\ \gamma & \text { otherwise }\end{cases}
$$

and define

$$
g_{\gamma, k}(\boldsymbol{x})=\operatorname{Sym}\left(\frac{x^{-k+1} \prod_{i \in I} \prod_{r=1}^{m_{i}}\left(x_{r}^{(i)}\right)^{-\left(\alpha_{i}, \gamma^{(i, r)}\right)} \cdot h_{\gamma}(\boldsymbol{x})}{\prod_{\substack{i<j \\ c_{i j}<0}} \prod_{r, s}\left(x_{r}^{(i)}-x_{s}^{(j)}\right)}\right)
$$

for $k \in \mathbb{Z}$, where Sym denotes the symmetrization over $n$ sets of variables $\left\{x_{r}^{(i)} \mid 1 \leq\right.$ $\left.r \leq m_{i}\right\}(i \in I)$, and $x \in \boldsymbol{x}_{\gamma}$ is an arbitrarily fixed variable. We easily check that $g_{\gamma, k}(\boldsymbol{x}) \in \mathcal{U}_{\gamma}$. Moreover, it follows from a direct calculation that

$$
\begin{aligned}
\operatorname{deg} h_{\gamma}(\boldsymbol{x}) & =\sum_{i \in I \backslash I_{\mathrm{sh}}} m_{i}\left(m_{i}-1\right)+\sum_{i \in I_{\mathrm{sh}}}\left\lceil\frac{m_{i}\left(m_{i}-2\right)}{2}\right\rceil+\sum_{\substack{i, j \in I_{\mathrm{sh}} ; \\
i<j, c_{i j}=-1}}\left\lfloor\frac{m_{i} m_{j}}{2}\right\rfloor \\
& =\frac{1}{2}\{(\gamma, \gamma)+(\bar{\gamma}, \bar{\gamma})\}-\operatorname{ht}(\gamma)+\sum_{\substack{i<j \\
c_{i j}<0}} m_{i} m_{j}=1-\operatorname{ht}(\gamma)+\sum_{\substack{i<j \\
c_{i j}<0}} m_{i} m_{j},
\end{aligned}
$$

and then we have by (4.4) that

$$
\left\langle f_{\gamma, k}, g_{\gamma, k}(\boldsymbol{x})\right\rangle \in \mathbb{C}^{\times}, \quad\left\langle f_{\gamma^{\prime}, k^{\prime}}, g_{\gamma, k}(\boldsymbol{x})\right\rangle=0 \text { if } \gamma-\gamma^{\prime} \notin Q^{+} \text {or } \gamma=\gamma^{\prime}, k \neq k^{\prime} .
$$

It is also proved from Lemma 4.6 that $g_{\gamma, k}(\boldsymbol{x}) \in \overline{\mathcal{U}}_{\gamma}$ if $k>0$.

When $\mathfrak{g}$ is of type $G_{2}$, define $g_{\gamma, k}(\boldsymbol{x}) \in \overline{\mathcal{U}}_{\gamma}$ for $\gamma \in R^{+}$and $k \in \mathbb{Z}_{>0}$ by

$$
g_{\gamma, k}(\boldsymbol{x})= \begin{cases}\left(x_{1}^{(i)}\right)^{-k-1} & \text { if } \gamma=\alpha_{i}, \\ \left(x_{1}^{(1)}\right)^{-k+m-1} \prod_{r=1}^{m}\left(x_{r}^{(2)}\right)^{-1} / \Delta_{\gamma} & \text { if } \gamma=\alpha_{1}+m \alpha_{2}, \\ \operatorname{Sym}\left(\left(x_{1}^{(1)}\right)^{-k}\left(x_{2}^{(1)}\right)^{-1}\left(x_{1}^{(1)}-x_{2}^{(1)}\right)^{2} / \Delta_{\gamma}\right) & \text { if } \gamma=2 \alpha_{1}+3 \alpha_{2},\end{cases}
$$

where $\alpha_{1}$ is long and $\alpha_{2}$ is short. We easily check that these $g_{\gamma, k}(\boldsymbol{x})$ also satisfy (A.3).

Now we show Proposition 4.7. The proof is carried out in a similar line as $\mathrm{FKL}^{+} 02$, Proposition 3.1.3], in which the case $\mathfrak{g}=\mathfrak{s l}_{3}$ is treated.

Proof of Proposition 4.X. The injectivity is proved as follows. Let $0 \neq g(\boldsymbol{x}) \in \overline{\mathcal{U}}_{\gamma}$, and consider it as a formal Laurent series by expanding all $\left(x_{r}^{(i)}-x_{s}^{(j)}\right)^{-1}(i<j)$ in positive power of $x_{s}^{(j)} / x_{r}^{(i)}$. If the coefficient of a monomial $\prod_{r}\left(x_{r}^{(1)}\right)^{k_{r}^{(1)}} \cdots \prod_{r}\left(x_{r}^{(n)}\right)^{k_{r}^{(n)}}$ in the series is $c \neq 0$, then taking $F=\prod_{r} f_{1,-k_{r}^{(1)}-1} \cdots \prod_{r} f_{n,-k_{r}^{(n)}-1}$, we have $\langle F, g(\boldsymbol{x})\rangle=c \neq$ 0 . Hence the injectivity is proved.

Next we prove that for any $F \in\left(\bar{U}_{\mathbf{L}}^{-}\right)_{-\gamma}$ there exists $g(\boldsymbol{x}) \in \overline{\mathcal{U}}_{\gamma}$ such that $\langle F, g(\boldsymbol{x})\rangle \neq$ 0 , which implies the surjectivity and completes the proof. We may assume $F \in U\left(t \mathfrak{n}_{-}[t]\right)$ by the Poincaré-Birkhoff-Witt theorem. Fix a total ordering $\preceq$ on $R^{+}$such that $\alpha \preceq \beta$ holds if $\beta-\alpha \in Q^{+}$. We also denote by $\preceq$ the lexicographic ordering on $R^{+} \times \mathbb{Z}_{>0}$. Let $B$ be the Poincaré-Birkhoff-Witt basis of $U\left(t \mathfrak{n}_{-}[t]\right)$ with respect to this ordering;

$$
B=\left\{f_{\beta_{N}, k_{N}} \cdots f_{\beta_{2}, k_{2}} f_{\beta_{1}, k_{1}} \mid \beta_{a} \in R^{+}, k_{a} \in \mathbb{Z}_{>0},\left(\beta_{N}, k_{N}\right) \preceq \cdots \preceq\left(\beta_{1}, k_{1}\right)\right\} .
$$


Write $F=\sum_{b \in B} c_{b} b$ with $c_{b} \in \mathbb{C}$, and let $b_{0}=f_{\beta_{N}, k_{N}} \cdots f_{\beta_{2}, k_{2}} f_{\beta_{1}, k_{1}} \in B$ be the minimum vector with respect to the right-to-left lexicographic order such that $c_{b_{0}} \neq 0$. Then define $g(\boldsymbol{x}) \in \overline{\mathcal{U}}_{\gamma}$ by

$$
g(\boldsymbol{x})=\operatorname{Sym}\left(\psi_{\beta_{<N}}\left(g_{\beta_{N}, k_{N}}\left(\boldsymbol{x}_{\beta_{N}}\right)\right) \cdots \psi_{\beta_{<2}}\left(g_{\beta_{2}, k_{2}}\left(\boldsymbol{x}_{\beta_{2}}\right)\right) \cdot g_{\beta_{1}, k_{1}}\left(\boldsymbol{x}_{\beta_{1}}\right)\right),
$$

where we set $\beta_{<a}=\beta_{1}+\cdots+\beta_{a-1}$, and $\psi_{\beta}$ for $\beta=\sum_{i} m_{i} \alpha_{i}$ to be the algebra homomorphism defined by $\psi_{\beta}\left(x_{r}^{(i)}\right)=x_{r+m_{i}}^{(i)}$. It is proved from (A.3) that

$$
\left\langle b_{0}, g(\boldsymbol{x})\right\rangle \in \mathbb{C}^{\times}, \quad\left\langle b^{\prime}, g(\boldsymbol{x})\right\rangle=0 \text { for } b^{\prime} \in B \text { such that } b^{\prime} \succ b_{0},
$$

and hence we have $\langle F, g(\boldsymbol{x})\rangle \neq 0$, as required. The proof is complete.

A.4. Proof of Lemma 4.8. Let $\lambda=\sum_{k} \ell_{k} \varpi_{i_{k}} \in P^{+}$and $\gamma=\sum_{i} m_{i} \alpha_{i} \in Q^{+}$be as in Lemma 4.8. Let $\boldsymbol{\mu}$ be an $I$-tuple of partitions such that $\left|\mu^{(i)}\right|=m_{i}$ and $\mu^{(i)}$ has $m_{a}^{(i)}$ rows of length $a$, and assume that $g(\boldsymbol{x}) \in \mathcal{V}_{\gamma} \cap \Gamma_{\boldsymbol{\mu}}$. By Lemma A.1 and the definition of $\mathcal{V}_{\gamma}$, the function $\varphi_{\boldsymbol{\mu}}(g(\boldsymbol{x}))$ is written as $\varphi_{\boldsymbol{\mu}}(g(\boldsymbol{x}))=h_{0}\left(\boldsymbol{y}_{\boldsymbol{\mu}}\right) h_{1}\left(\boldsymbol{y}_{\boldsymbol{\mu}}\right)$, where

$$
h_{0}\left(\boldsymbol{y}_{\boldsymbol{\mu}}\right)=\frac{\prod_{i \in I} \prod_{(a, u)<(b, v)}\left(y_{a, u}^{(i)}-y_{b, v}^{(i)}\right)^{2 \min \{a, b\}}}{\prod_{i \in I} \prod_{(a, u)}\left(y_{a, u}^{(i)}\right)^{\sum_{k \in S_{i}} \min \left\{a, \ell_{k}\right\}} \prod_{i<j} \prod_{(a, u),(b, v)}\left(y_{a, u}^{(i)}-y_{b, v}^{(j)}\right)^{\min \left\{\left|c_{j i}\right| a,\left|c_{i j}\right| b\right\}}},
$$

and $h_{1}\left(\boldsymbol{y}_{\boldsymbol{\mu}}\right)$ is a polynomial symmetric under the exchange $y_{a, u}^{(i)} \leftrightarrow y_{a, v}^{(i)}$. Moreover by Lemma 4.6, we have

$$
\operatorname{deg}_{y_{a, u}^{(i)}} \varphi_{\boldsymbol{\mu}}(g(\boldsymbol{x})) \leq-2 a
$$

for all $i, a, u$. Since $\operatorname{deg}_{y_{a, u}^{(i)}} h_{0}\left(\boldsymbol{y}_{\boldsymbol{\mu}}\right)=-p_{a}^{(i)}-2 a$, we have $\operatorname{deg}_{y_{a, u}^{(i)}} h_{1}\left(\boldsymbol{y}_{\boldsymbol{\mu}}\right) \leq p_{a}^{(i)}$ for all $i, a, u$. Hence it follows that $\varphi_{\boldsymbol{\mu}}\left(\mathcal{V}_{\gamma} \cap \Gamma_{\boldsymbol{\mu}}\right)=0$ if $p_{a}^{(i)}<0$ for some $i, a$, and otherwise

$$
\operatorname{dim} \varphi_{\boldsymbol{\mu}}\left(\mathcal{V}_{\gamma} \cap \Gamma_{\boldsymbol{\mu}}\right) \leq \prod_{\substack{i \in I \\
a>0}}\left(\begin{array}{c}
p_{a}^{(i)}+m_{a}^{(i)} \\
m_{a}^{(i)}
\end{array}\right)
$$

Since $\varphi_{\boldsymbol{\mu}}\left(\mathcal{V}_{\gamma} \cap \Gamma_{\boldsymbol{\mu}}\right) \cong\left(\mathcal{V}_{\gamma} \cap \Gamma_{\boldsymbol{\mu}}\right) /\left(\mathcal{V}_{\gamma} \cap \Gamma_{\boldsymbol{\mu}}^{\prime}\right)$ and

$$
\operatorname{dim} \mathcal{V}_{\gamma}=\sum_{\boldsymbol{\mu}} \operatorname{dim}\left(\mathcal{V}_{\gamma} \cap \Gamma_{\boldsymbol{\mu}}\right) /\left(\mathcal{V}_{\gamma} \cap \Gamma_{\boldsymbol{\mu}}^{\prime}\right),
$$

the lemma follows.

\section{ACKNOWLEDGMENTS}

The author is supported by JSPS Grant-in-Aid for Young Scientists (B) No. 25800006.

\section{REFERENCES}

[AK07] E. Ardonne and R. Kedem. Fusion products of Kirillov-Reshetikhin modules and fermionic multiplicity formulas. J. Algebra, 308(1):270-294, 2007.

[AKS06] E. Ardonne, R. Kedem, and M. Stone. Fermionic characters and arbitrary highest-weight integrable $\widehat{\mathfrak{s l}}_{r+1}$-modules. Comm. Math. Phys., 264(2):427-464, 2006.

[BCM15] M. Brito, V. Chari, and A. Moura. Demazure modules of level two and prime representations of quantum affine $\mathfrak{s l}_{n+1}$. J. Inst. Math. Jussieu, 31 pages, 2015. 
[Bec94] J. Beck. Braid group action and quantum affine algebras. Comm. Math. Phys., 165(3):555568, 1994.

[BP15] M. Brito and F. Pereira. Graded limits of simple tensor product of Kirillov-Reshetikhin modules for $U_{q}\left(\widetilde{\mathfrak{s l}}_{n+1}\right)$. arXiv:1504.05907.

[CH10] V. Chari and D. Hernandez. Beyond Kirillov-Reshetikhin modules. In Quantum affine algebras, extended affine Lie algebras, and their applications, volume 506 of Contemp. Math., pages 49-81. Amer. Math. Soc., Providence, RI, 2010.

[Cha01] V. Chari. On the fermionic formula and the Kirillov-Reshetikhin conjecture. Int. Math. Res. Not. IMRN, (12):629-654, 2001.

[Cha02] V. Chari. Braid group actions and tensor products. Int. Math. Res. Not., (7):357-382, 2002.

[CI15] V. Chari and B. Ion. BGG reciprocity for current algebras. Compos. Math., 151(7):1265$1287,2015$.

[CL06] V. Chari and S. Loktev. Weyl, Demazure and fusion modules for the current algebra of $\mathfrak{s l}_{r+1}$. Adv. Math., 207(2):928-960, 2006.

[CM06] V. Chari and A. Moura. The restricted Kirillov-Reshetikhin modules for the current and twisted current algebras. Comm. Math. Phys., 266(2):431-454, 2006.

[CP91] V. Chari and A. Pressley. Quantum affine algebras. Comm. Math. Phys., 142(2):261-283, 1991.

[CP94] V. Chari and A. Pressley. A guide to quantum groups. Cambridge University Press, Cambridge, 1994.

[CP95] V. Chari and A. Pressley. Quantum affine algebras and their representations. In Representations of groups (Banff, AB, 1994), volume 16 of CMS Conf. Proc., pages 59-78. Amer. Math. Soc., Providence, RI, 1995.

[CP96] V. Chari and A. Pressley. Minimal affinizations of representations of quantum groups: the simply laced case. J. Algebra, 184(1):1-30, 1996.

[CP01] V. Chari and A. Pressley. Weyl modules for classical and quantum affine algebras. Represent. Theory, 5:191-223 (electronic), 2001.

[CV15] V. Chari and R. Venkatesh. Demazure modules, fusion products and $Q$-systems. Comm. Math. Phys., 333(2):799-830, 2015.

[DFK08] P. Di Francesco and R. Kedem. Proof of the combinatorial Kirillov-Reshetikhin conjecture. Int. Math. Res. Not. IMRN, (7):Art. ID rnn006, 57, 2008.

[Dri87] V. G. Drinfel'd. A new realization of Yangians and of quantum affine algebras. Dokl. Akad. Nauk SSSR, 296(1):13-17, 1987.

[FF02] B. Feigin and E. Feigin. Q-characters of the tensor products in $\mathfrak{s l}_{2}$-case. Mosc. Math. J., 2(3):567-588, 2002. Dedicated to Yuri I. Manin on the occasion of his 65th birthday.

[FH14] G. Fourier and D. Hernandez. Schur positivity and Kirillov-Reshetikhin modules. SIGMA Symmetry Integrability Geom. Methods Appl., 10:Paper 058, 9, 2014.

$\left[\mathrm{FKL}^{+} 02\right]$ B. Feigin, R. Kedem, S. Loktev, T. Miwa, and E. Mukhin. Combinatorics of the $\widehat{\mathfrak{s l}}_{2}$ coinvariants: dual functional realization and recursion. Compositio Math., 134(2):193-241, 2002.

[FL99] B. Feigin and S. Loktev. On generalized Kostka polynomials and the quantum Verlinde rule. In Differential topology, infinite-dimensional Lie algebras, and applications, volume 194 of Amer. Math. Soc. Transl. Ser. 2, pages 61-79. Amer. Math. Soc., Providence, RI, 1999.

[FL07] G. Fourier and P. Littelmann. Weyl modules, Demazure modules, KR-modules, crystals, fusion products and limit constructions. Adv. Math., 211(2):566-593, 2007.

[FM01] E. Frenkel and E. Mukhin. Combinatorics of $q$-characters of finite-dimensional representations of quantum affine algebras. Comm. Math. Phys., 216(1):23-57, 2001.

[FS94] B. Feigin and A. Stoyanovsky. Functional models of the representations of current algebras, and semi-infinite Schubert cells. Funktsional. Anal. i Prilozhen., 28(1):68-90, 96, 1994.

[Her06] D. Hernandez. The Kirillov-Reshetikhin conjecture and solutions of T-systems. J. Reine Angew. Math., 596:63-87, 2006.

$\left[\mathrm{HKO}^{+} 99\right]$ G. Hatayama, A. Kuniba, M. Okado, T. Takagi, and Y. Yamada. Remarks on fermionic formula. In Recent developments in quantum affine algebras and related topics (Raleigh, NC, 
1998), volume 248 of Contemp. Math., pages 243-291. Amer. Math. Soc., Providence, RI, 1999.

[Kac90] V.G. Kac. Infinite-dimensional Lie algebras. Cambridge University Press, Cambridge, third edition, 1990.

[Ked11] R. Kedem. A pentagon of identities, graded tensor products, and the Kirillov-Reshetikhin conjecture. In New trends in quantum integrable systems, pages 173-193. World Sci. Publ., Hackensack, NJ, 2011.

[KR87] A. N. Kirillov and N. Yu. Reshetikhin. Representations of Yangians and multiplicities of the inclusion of the irreducible components of the tensor product of representations of simple Lie algebras. Zap. Nauchn. Sem. Leningrad. Otdel. Mat. Inst. Steklov. (LOMI), 160(Anal. Teor. Chisel i Teor. Funktsii. 8):211-221, 301, 1987.

[LLM02] V. Lakshmibai, P. Littelmann, and P. Magyar. Standard monomial theory for Bott-Samelson varieties. Compositio Math., 130(3):293-318, 2002.

[LN16] J. R. Li and K. Naoi. Graded limits of minmal affinizations over the quantum loop algebra of type $G_{2}$. Algebr. Represent. Theory, 17 pages, 2016.

[Lus89] G. Lusztig. Affine Hecke algebras and their graded version. J. Amer. Math. Soc., 2(3):599635, 1989.

[Lus90] G. Lusztig. Quantum groups at roots of 1. Geom. Dedicata, 35(1-3):89-113, 1990.

[Lus93] G. Lusztig. Introduction to quantum groups, volume 110 of Progress in Mathematics. Birkhäuser Boston Inc., Boston, MA, 1993.

[Mou10] A. Moura. Restricted limits of minimal affinizations. Pacific J. Math., 244(2):359-397, 2010.

[Nak03] H. Nakajima. $t$-analogs of $q$-characters of Kirillov-Reshetikhin modules of quantum affine algebras. Represent. Theory, 7:259-274 (electronic), 2003.

[Nao12a] K. Naoi. Fusion products of Kirillov-Reshetikhin modules and the $X=M$ conjecture. Adv. Math., 231(3-4):1546-1571, 2012.

[Nao12b] K. Naoi. Weyl modules, Demazure modules and finite crystals for non-simply laced type. Adv. Math., 229(2):875-934, 2012.

[Nao13a] K. Naoi. Demazure crystals and tensor products of perfect Kirillov-Reshetikhin crystals with various levels. J. Algebra, 374:1-26, 2013.

[Nao13b] K. Naoi. Demazure modules and graded limits of minimal affinizations. Represent. Theory, $17: 524-556,2013$.

[Nao14] K. Naoi. Graded limits of minimal affinizations in type D. SIGMA Symmetry Integrability Geom. Methods Appl., 10:Paper 047, 20 pages, 2014.

[Nao15] K. Naoi. Defining relations of fusion products and schur positivity. Toyama Math. J., 37:87$106,2015$.

[OS08] M. Okado and A. Schilling. Existence of Kirillov-Reshetikhin crystals for nonexceptional types. Represent. Theory, 12:186-207, 2008.

Institute of Engineering, Tokyo University of Agriculture And Technology, 2-24-16 NAKA-CHO, KOGANEI-SHI, TOKYO 184-8588, JAPAN

E-mail address: naoik@cc.tuat.ac.jp 